\title{
La prévention des inondations dans les opérations d'aménagement des interfaces ville-port, un levier de gentrification indirecte? Le cas du Havre (France)
}

Florence Orillard, Mathilde Gralepois et Laura Verdelli

\section{OpenEdition}

\section{Journals}

Édition électronique

URL : https://journals.openedition.org/cdg/1713

DOI : $10.4000 /$ cdg. 1713

ISSN : 2107-7266

\section{Éditeur}

UMR 245 - CESSMA

\section{Référence électronique}

Florence Orillard, Mathilde Gralepois et Laura Verdelli, « La prévention des inondations dans les opérations d'aménagement des interfaces ville-port, un levier de gentrification indirecte? Le cas du Havre (France) ", Carnets de géographes [En ligne], 11 | 2018, mis en ligne le 15 septembre 2018, consulté le 20 mai 2021. URL : http://journals.openedition.org/cdg/1713 ; DOI : https://doi.org/ $10.4000 /$ cdg. 1713

Ce document a été généré automatiquement le 20 mai 2021.

\section{c)}

La revue Carnets de géographes est mise à disposition selon les termes de la Licence Creative Commons Attribution - Pas d'Utilisation Commerciale - Pas de Modification 4.0 International. 


\title{
La prévention des inondations dans les opérations d'aménagement des interfaces ville-port, un levier de gentrification indirecte? Le cas du Havre (France)
}

\author{
Florence Orillard, Mathilde Gralepois et Laura Verdelli
}

\section{Introduction}

$1 \quad$ Les projets urbains de waterfront cities se développent depuis les années 1970 dans les villes nord-américaines (Baltimore, San Francisco, Boston...) et s'accélèrent à partir des années 1990 dans les villes-ports de l'Europe continentale (Hambourg, Lisbonne, Barcelone, Angers, Amsterdam.... $)^{1}$. Le projet urbain devient symbole de dynamisme et de puissance pour les décideurs publics. L'enjeu est de renouveler des espaces précédemment dédiés aux activités portuaires ou abritant des logements dégradés. Dans les villes européennes où l'activité du port fut directement liée aux activités commerciales des centres-villes, ces espaces sont aujourd'hui proches d'une partie des centralités et bénéficient d'une bonne accessibilité. L'enjeu est de ramener ces quartiers portuaires au cœur des villes, et de les y rattacher pour en faire des espaces résidentiels, tertiaires et touristiques. Dans ces lieux, la puissance du projet d'aménagement à porter un message de transformation économique et social est particulièrement visible.

2 Le projet urbain (urban design), à l'instar de la planification stratégique (urban planning), se définit souvent en cours de réalisation ${ }^{2}$, par la participation de tous les acteurs aux différents stades de formulation (à la fois les acteurs traditionnels de l'aménagement urbain, mais aussi de plus en plus d'acteurs associatifs, privés, habitants...), permettant une prise en compte de la complexité urbaine et des attentes de la population (Bourdin, 
2001 ; Ingallina, 2001; Novarina, 2003). Concrètement, le projet urbain est appelé à stimuler l'économie locale lors de la période des études et du chantier de construction, mais aussi à redévelopper l'économie résidentielle et présentielle par le développement de logements et l'implantation de services. De plus, le processus de projet à l'échelle du quartier permet aux municipalités de projeter une vision politique visant à faire évoluer plus ou moins radicalement les représentations. En jouant sur des facteurs symboliques, l'aménagement d'un quartier transforme l'histoire, l'image, l'avenir, mais aussi la population.

3 Les récents projets de requalification des espaces portuaires proposent une offre de logements et de services dédiés à une population relativement aisée, ce qui contraste avec les études statistiques (Chesnel, 2015) qui constatent une forte présence de populations souvent en situation économique et sociale précaire. De ce fait, ces projets encouragent le processus de gentrification dans sa signification large, c'est-à-dire plus généralement un phénomène socio-spatial d'élitisation (Rérat et al., 2008), connu pour s'accompagner d'une requalification d'image (Rousseau, 2014). Nous montrerons qu'il ne s'agit pas uniquement d'une stratégie de mixité sociale, mais bien d'une transformation - disons même un remplacement - à la fois de l'image, de l'histoire, du patrimoine, des activités économiques, des fonctions urbaines et aussi de la population. L'usage du terme gentrification s'est largement étendu et intègre aujourd'hui des processus et des acteurs clefs qui se sont diversifiés. En accord avec l'argumentaire porté par Davidon et Lees (2005) sur l'évolution contemporaine de ce concept ${ }^{3}$, cet article s'appuie sur une définition de la gentrification qui intègre les nouveaux développements résidentiels prenant place dans les friches urbaines et portuaires, ou new build gentrification (Davidson et Lees, 2005).

4 Les villes françaises opérant cette politique de gentrification des lieux, qui passe par l'amélioration de l'image du quartier et impacte la composition sociale de la population, font face à une implication forte de la société civile sous forme de contestations, comme à Marseille avec le quartier de la Joliette (Jourdan, 2008), ou le quartier d'Arenc (Prelorenzo, 2010) ou à Lyon avec le projet Confluences (Adam, 2016). La contestation sociale est considérée par les autorités locales comme génératrice de surcoûts, de délais et d'une perte d'efficacité dans les projets de territoire, ce qui induit une recherche de moyens visant à éviter de telles confrontations.

Dans le cas des quartiers industrialo-portuaires, un moyen de contournement de cette mobilisation semble émerger: l'intégration des mesures de prévention des risques majeurs ${ }^{4}$. En effet, la situation maritime ou estuarienne des grands ports induit la présence d'un risque d'inondation qui peut se manifester par un risque de submersion marine, de débordement des cours d'eau et des réseaux, de remontée de nappes affleurantes ou de phénomènes d'inondation conjuguant ces facteurs. Lors de la construction d'un quartier en zone inondable, comment sont mobilisées les contraintes géographiques et les implications juridiques liées au risque majeur? Quelles sont les conséquences sociales de la prise en compte des règles appliquées aux territoires à risque d'inondation? Tel que pressenti par Rode (2008) à Blois, nous analysons comment le projet d'aménagement urbain d'un quartier portuaire au Havre (France) engendre des effets de gentrification dans les quartiers renouvelés dans des espaces à risque d'inondation.

6 Dans la lignée des analyses sur la relation géopolitique entre les risques naturels et les territoires (Pigeon, 2015; Reghezza-Zitt, 2015), l'étude des effets de la gestion 
territorialisée du risque d'inondation vient compléter l'étude des processus géographiques et politiques de la gentrification. L'article enrichit la littérature sur les opérations d'aménagement dans les espaces inondables constructibles, en prenant le cas de l'espace industrialo-portuaire du Havre. Les quartiers Sud situés entre la ville et les espaces portuaires en activité sont un espace d'enjeux majeur pour le développement d'une interface ville/port, à l'image des grands ports européens. L'extrémité Ouest des quartiers Sud, traditionnellement nommée "Quartier de l'Eure » mais promue par le projet de revitalisation sous le nom «Saint Nicolas », est la partie la plus proche du centre-ville: elle abrite des friches industrialo-portuaires mais également quelques petits îlots urbains dégradés. Le projet urbain transforme radicalement, en l'espace de 10 ans, un espace industrialo-portuaire en quartier résidentiel à fort potentiel économique à travers la reconnexion avec le centre-ville (gare, tramway...), la requalification des espaces publics (parc urbain, accessibilité automobile, visibilité sur les bassins, utilisation des symboles portuaires...) et des logements d'architecture contemporaine.

7 L'article montre comment s'opère, dans un contexte de reconquête de friches industrialo-portuaires, l'instrumentalisation des contraintes liées au risque inondation pour soutenir un processus, voire une stratégie, de gentrification. En effet, la prévention des risques naturels dans les projets de renouvellement urbain participe - par un discours de sécurité collective - à justifier l'usage des outils classiques de gentrification utilisés par les projets urbains ${ }^{5}$.

8 L'hypothèse est mise à l'épreuve via les informations collectées dans le cadre d'un projet de recherche ${ }^{6}$, d'un stage de fin d'étude ${ }^{7}$ et d'un mémoire de fin d'étude ${ }^{8}$. La méthode utilisée est essentiellement fondée sur l'analyse des documents réglementaires, des stratégies d'aménagement, des plans opérationnels d'urbanisme et des discours affichés qui les accompagnent. Une douzaine d'entretiens semi-directifs avec les acteurs locaux de l'aménagement et de la prévention des risques ${ }^{9}$ permet comprendre les contextes de réalisation et les intentions concrètes de l'action. Enfin, les chercheurs $\mathrm{du}$ projet ont réalisé plusieurs visites de terrain, certaines accompagnées de professionnels locaux notamment au Havre en mars 2015 avec un représentant de l'Agence d'Urbanisme de la Région Havraise.

9 L'article présente, dans un premier temps, les différentes stratégies de requalification des waterfronts illustrées par celles mises en place au Havre. Dans un second temps, il présente plus en détail le processus de requalification des espaces portuaires des quartiers Sud du Havre, montrant que la prise en compte des risques d'inondation favorise implicitement un processus de gentrification. L'exemple havrais amène enfin à s'interroger sur une possible généralisation: la prise en compte des risques d'inondation peut-elle produire un urbanisme sélectif?

\section{Les stratégies de requalification des interfaces ville- port}

10 La diminution du volume des finances publiques locales place les villes en déséquilibre économique (Cour des comptes, 2014 ; Rapport du Sénat, 2014). Pour gérer la pression budgétaire, les municipalités tentent d'agir sur leurs sources de revenu, notamment en élargissant l'assiette de la fiscalité locale et en rentabilisant leurs opérations urbaines. Cette logique traditionnellement associée au domaine privé implique la puissance 
publique dans la spéculation foncière des espaces considérés comme économiquement valorisables (Bachimon, 2014). Face à la crise des villes industrielles européennes, le besoin de relance économique passe par des dynamiques basées sur le développement résidentiel, portées par deux actions de requalification: le remplacement de l'image patrimoniale et paysagère antérieure du quartier et la requalification urbaine des zones délaissées et pourtant valorisables telles que des interfaces villes-ports.

\section{La production d'une image attractive pour le développement de l'économie résidentielle et présentielle : le cas du Havre}

11 Frappées par la désindustrialisation des années 1970, les dynamiques économiques du Havre sont mises en concurrence avec les ports nord-européens depuis les années 1990. Pourtant, l'économie havraise est entièrement tournée vers son port, qui reste le $6^{\text {ème }}$ port européen et le $1^{\mathrm{er}}$ port français pour le transport de conteneurs. Son activité industrielle et commerciale irrigue la ville de 30190 emplois (INSEE, 2015), dont 13470 dans le cluster maritime et portuaire et 16720 dans le cluster industriel. Le dynamisme économique havrais continue de porter en bonne partie sur "la prépondérance des industries lourdes, l'orientation des services vers les transports" (INSEE, 2006), et l'augmentation des flux permet de compenser les pertes du cluster industriel ${ }^{10}$. Or, l'incertitude économique liée à la concurrence et l'interchangeabilité des espaces de flux entraîne une instabilité des revenus. Alors les acteurs des politiques locales cherchent à attirer et ancrer ces flux sur le territoire (Veltz, 2008), notamment par le renforcement des aménités locales qu'elles soient sociales, environnementales, culturelles ou symboliques.

12 En parallèle, le déclin démographique de la ville du Havre ne s'inverse pas depuis 1975, date à laquelle la population s'élevait à 218000 habitants. Passant sous la barre des 200000 dans les années 1980, la population est d'environ 177000 en 1999 puis d'environ 172000 habitants en 2014 (INSEE, 2014). De plus, la population ayant une propension locale à consommer tend à s'établir dans les communes périphériques situées autour du Havre (AURH, 2017: 5). Le Havre est marqué par un faible «couplage entre bassin d'habitat et bassin d'emplois » (Cusin, 2008), une faiblesse des revenus liés à la fiscalité locale, un manque d'animation et de vitalité commerciale, une "tertiarisation très lente de cette économie en général " (INSEE, 2006). Autant de signaux qui génèrent un faible potentiel d'attractivité de la ville. Pour stimuler l'attractivité auprès des entreprises et des populations, et parallèlement pour changer l'image du ville-centre, les politiques locales havraises - comme dans d'autres villes françaises en déclin telles que Saint-Etienne, Brest ou Caen - orientent radicalement leurs politiques de développement " autour d'un agenda centré sur l'urbanisme, le logement et les espaces publics, dont l'objectif central est de renforcer l'attractivité de la ville » (Cauchi-Duval, Béal, Rousseau, 2016). Depuis les années 2010, Le Havre affiche plusieurs symboles d'un modèle métropolitain tels que des moyens de transport rapides (mise en service des deux lignes de tramway en 2012), des équipements sportifs et culturels (à l'image du stade Océane de 2012 et de la rénovation récente du théâtre du Volcan en 2015), mais aussi de grands projets de ville (convention ANRU en 2004 et inscription UNESCO en 2005). En matière de projet urbain, la revitalisation des espaces industrialo-portuaires fait figure de symbole fort. 
Si l'économie des villes-ports repose largement sur le secteur secondaire, l'enjeu actuel de la ville du Havre est de valoriser les atouts de l'économie résidentielle et présentielle ${ }^{11}$. Au Havre, sans entrer plus avant dans la définition de termes propres aux débats de l'économie régionale, la stratégie de complétion des revenus liés à l'économie portuaire opère via le développement de politiques urbaines. Ces politiques visent à attirer une part de la population ayant une propension locale à consommer, au premier rang de laquelle les retraités, les touristes et les actifs des couches supérieures. Leur présence entraîne une circulation de revenus ayant pour partie vocation à être dépensées sur place et ainsi à stimuler la demande locale (Cusin, 2008). Pour attirer ces nouvelles populations, l'atout mis en avant dans les politiques urbaines du Havre porte sur la valorisation de la localisation maritime et fluviale de la ville. Cet objectif entraîne un remplacement de l'image du quartier, passant d'un quartier portuaire d'habitat déqualifié à un espace de centralités urbaines haut de gamme.

Comme la sphère productive havraise associée à la logistique et à l'industrie induit un déficit d'image vis-à-vis de la population capable de consommer, les stratégies des politiques locales havraises des années 2000-2010 témoignent d'un choix de communication unanimement tourné vers les activités maritimes à forte valeur symbolique et d'une utilisation massive des projets urbains en lien avec la mer pour relancer une dynamique économique. Plusieurs actions de communication montrent le positionnement de la ville du Havre dans une fonction de 'porte d'entrée maritime' vers Paris, comme les discours sur 'Normandy Avenue' portés par les agences de développement normandes (2006), comme le projet d'Antoine Grumbach 'Seine Métropole' (2009) lors du concours du Grand Paris, ou l'étude 'Seine Gateway ${ }^{\otimes ’}$ (2012). Plus précisément, les projets urbains havrais s'emparent du port comme d'un élément identitaire. Le réinvestissement massif du port par les politiques urbaines débute notamment par les quartiers industrialo-portuaires, au Sud de la ville, à une encablure de la gare et de l'université, quartiers pourtant considérés comme enclavés (Boquet, 2009) (Figure 1). La construction du centre commercial des Docks Vauban (2009) lance la revitalisation du quartier de l'Eure qui fait aujourd'hui «figure emblématique du renouveau havrais » (Ville du Havre, 2018).

Figure 1 : Extension par étape de la centralité vers les quartiers sud

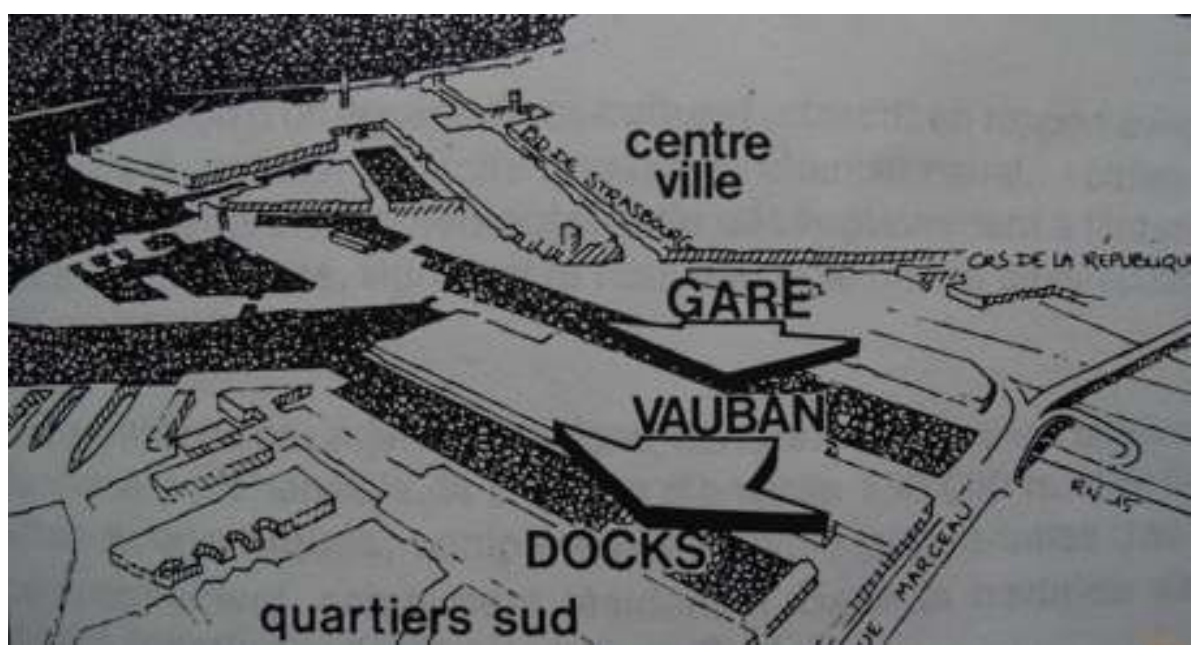

Source : Vauban Gare, Schéma d'aménagement directeur, division aménagement et urbanisme de la Ville du Havre, juin 1991 
Le site internet de la Ville du Havre décrit le quartier de l'Eure :

«Situé à l'interface entre la ville et le port, ce quartier populaire connaît depuis quelques années un nouvel essor grâce à des aménagements ambitieux qui concilient empreintes du passé et modernité. Le processus de reconversion engagé depuis plus de dix ans fait de ce quartier le théâtre de grands projets d'aménagement. » (Ville du Havre, 2018).

16 En engageant les termes d'interface, de quartier populaire ou de 'passé et modernité', les politiques urbaines havraises définissent des «avantages concurrentiels défendables » (Porter, 1985), tel que le patrimoine (Verdelli, 2014), qui préfigure le discours de marketing territorial ${ }^{12} \mathrm{du}$ projet urbain de l'Eure. Mais l'action sur un espace ne suffit plus, il faut en faire la promotion auprès d'un public préalablement ciblé. Pour rompre avec son déclin démographique et avec la fuite des foyers à hauts revenus de la ville, Le Havre investit les anciens espaces portuaires dans une démarche de mise en scène du patrimoine portuaire et urbain, en construisant un discours fédérateur qui réfléchirait les représentations attachées au port industriel afin d'amener de nouvelles sources de revenus résidentiels et présentiels (retraités, touristes, actifs des couches supérieures...). Cette action de communication participe d'une requalification urbaine 'par le haut' qui, nous allons le voir plus tard, va appuyer sa justification en partie sur le respect des normes de construction en zone inondable.

\section{La stratégie de requalification urbaine 'par le haut' des quartiers Sud du Havre}

17 La mécanisation des opérations portuaires et la course au gigantisme des conteneurs se traduisent par la libération d'un fort potentiel foncier à l'interface entre la ville et le port. Depuis les années 1990, la ville du Havre rachète des terrains au Grand Port Maritime du Havre ${ }^{13}$ pour réaliser nombre de projets urbains qui opère une requalification des quartiers portuaires. Or, les quartiers portuaires, notamment le quartier de l'Eure, sont touchés par l'existence d'un multirisque d'inondation (Figure 2).

Figure 2 : Le quartier de l'Eure inscrit dans un système multiple de contraintes d'inondation

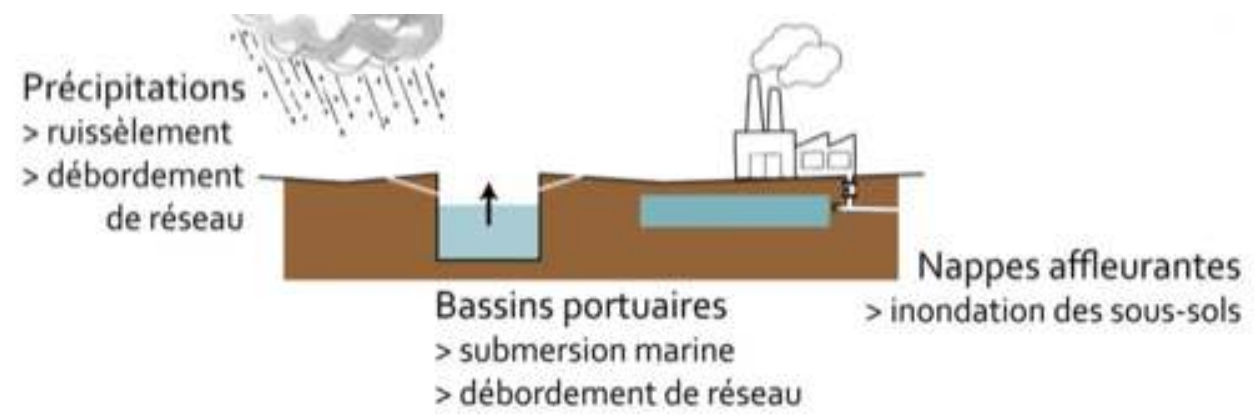

Source : Orillard, 2016

Implantée dans l'estuaire de la Seine et à faible altimétrie, la ville du Havre est historiquement concernée par le risque d'inondation par submersion marine. Ensuite, les fortes précipitations hivernales ou estivales auxquelles la ville est soumise génèrent également un risque d'inondation par le débordement des cours d'eau sur le plateau, le 
ruissellement urbain et/ou le débordement des réseaux urbains. Enfin, le sol marécageux de la ville basse induit un risque d'inondation par les variations des niveaux d'eau des nappes affleurantes. Si les risques d'inondation liés au débordement et au ruissellement du Bassin de la Lézarde sont pris en compte dans l'aménagement du Nord de l'agglomération du Havre par une planification des risques (Plan de prévention des risques de la Lézarde, révisé et approuvé en mai 2013), la prise en compte de l'inondation des espaces centraux havrais est au stade d'études et travaux. En 2014, dans le cadre de la transcription des directives européennes sur l'inondation dans le droit français ${ }^{14}$, le territoire du Havre élabore de nouvelles cartes d'aléas inondation intégrant les aléas débordement, ruissellement et les zones de submersion marine. Les cartes des surfaces inondables tenant compte de la submersion marine datent de mars 2016 (DREAL Normandie, 2016) (Figure 3). Pourtant, l'histoire havraise est marquée par des événements d'inondation tragiques, comme en 2003, et par le développement d'une politique active de prévention et de gestion des risques (Gralepois, 2008).

Figure 3 : Un risque de submersion désormais cartographié dans la zone portuaire du Havre

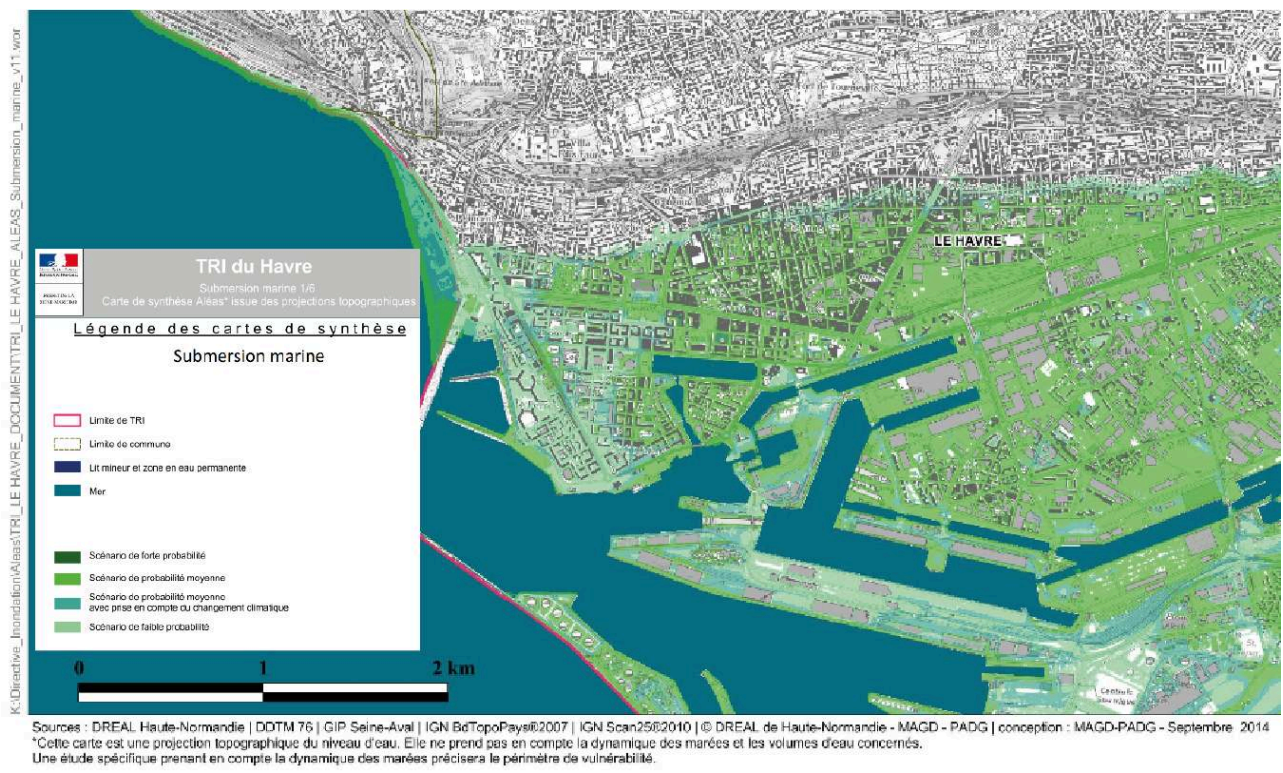

Source : Annexe II - Cartographie des surfaces inondables et des risques, TRI du Havre www.normandie.developpement-durable.gouv.fr/IMG/pdf/

TRI_Le_Havre_cartes_SM_synthese_cle6654a1.pdf (modifié par Orillard, 2018)

Même s'il n'existe pas encore en 2010 de document réglementaire permettant de maittriser l'urbanisme dans les zones de requalification industrialo-portuaire, un accord local met un place un double système de contrainte lors du développement du quartier de l'Eure. D'une part, un cahier de prescriptions architecturales et paysagères, réalisée par l'Assistance à Maîtrise d'Ouvrage (agence Bruno Fortier-Bloch) donne des recommandations pour tous les opérateurs privés. D'autre part, plus réglementairement, les annexes sanitaires au Plan Local d'Urbanisme imposent, pour simplifier, une surélévation du bâti et une interdiction de présence permanente de personnes dans les espaces souterrains.

20 Avant de montrer spécifiquement le rôle que la prise en compte des risques d'inondation dans la gentrification des quartiers industrialo-portuaires du Havre joue en tant qu'effet disculpant la 'montée en gamme' du quartier, il faut expliquer la stratégie 
de requalification des friches portuaires du Havre, en zoomant sur les opérations du quartier de l'Eure. Les quartiers Sud du Havre ont abrité jusqu'à la fin des années 1970 de nombreuses activités portuaires et industrielles qui ont laissées un contexte urbain dégradé et pollué, abritant une population qui s'est progressivement précarisée. Au global, la stratégie du projet de l'Eure n'est pas de faire table rase du passé portuaire et industriel (Jourdan, 2008), mais de patrimonialiser certaines composantes paysagères considérées comme valorisables auprès de nouvelles populations à attirer.

L'objectif du projet urbain, dont les principales opérations sont concentrées à l'extrémité Ouest des quartiers étudiés est double : réintégrer des fonctions de centreville et valoriser le patrimoine.

"Ce quartier a été pensé comme une extension du centre ville avec des équipements à l'échelle de l'agglomération, de la région, et même plus. » (Service permis de construire, Ville du Havre, juin 2017)

La stratégie du projet urbain consiste à requalifier les espaces délaissés par le port, et à miser sur la proximité de la gare, du tramway ou encore de l'Université pour y étendre les fonctions de centralité (Figure 4).

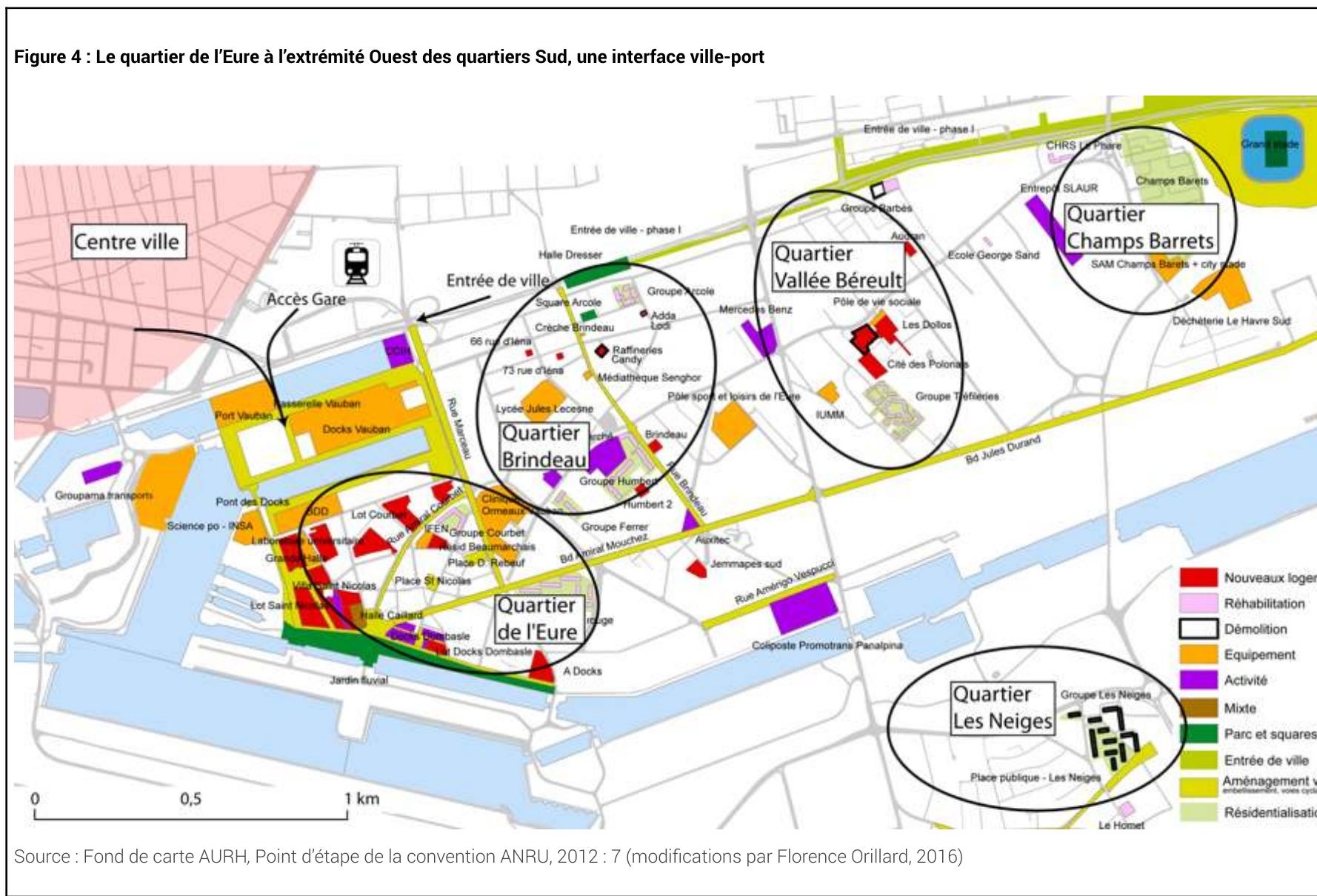

Le projet urbain de l'Eure s'oriente vers trois ambitions générales pour revaloriser l'attractivité du quartier: développer une offre culturelle et de loisirs autour de la thématique de l'eau (exemple: Bain des Docks), requalifier le paysage portuaire (exemple : Jardin Fluvial) et renouveler l'offre de logement (exemple : Docks Dombasle) (Figure 5). 
Figure 5 : Frise chronologique des opérations emblématiques du projet urbain du quartier de l'Eure

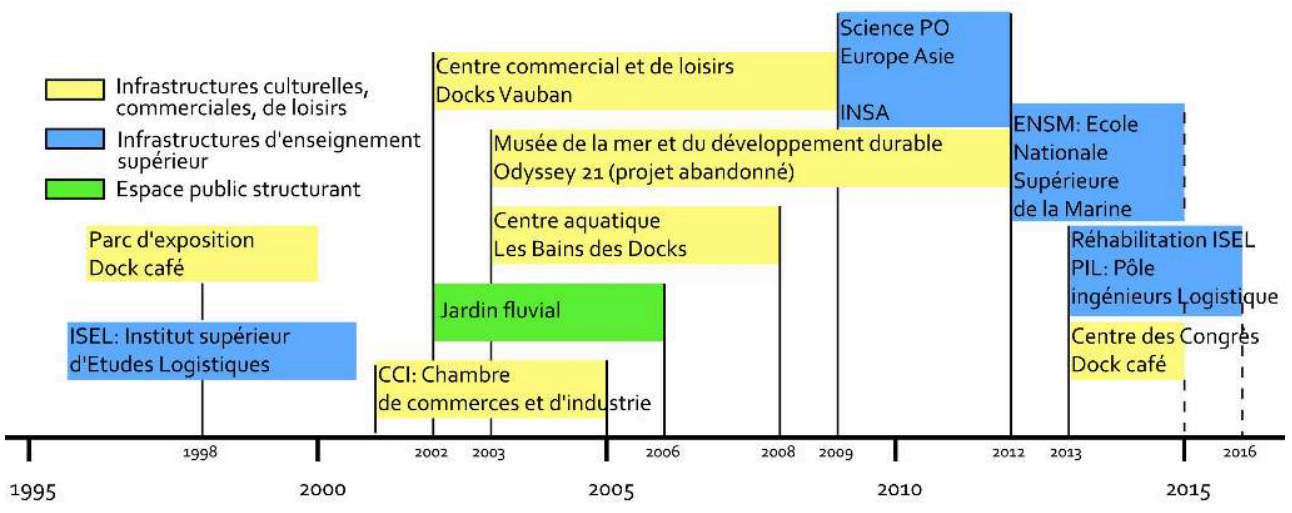

Source : Orillard, 2016

les opérations-phare de loisirs, on retrouve le Bain des Docks. Inauguré en 2008 il s'agit d'un complexe aquatique dont la maîtrise d'ouvrage a été porté la Mairie du Havre et la Communauté d'agglomération du Havre, comme pour la majorité des équipements et des espaces publics du quartier de l'Eure. Après concours, c'est l'atelier Jean Nouvel qui est nommé chef de projet. La construction a duré de 2004 à 2008, pour un coût total de 30 Millions d'euros. Pour cette réalisation, l'Atelier Jean Nouvel met l'accent sur la relation entre l'eau du bassin Vatine et l'eau du centre aquatique, ainsi que sur l'intégration du bâtiment dans le style architectural sobre du bâti portuaire :

«Imaginés comme un épaississement du quai, les Bains des docks se présentent aux visiteurs tels une masse évidée. Un jeu d'ouverture aléatoire sur les façades donne à voir depuis l'extérieur la richesse des espaces intérieurs habités par l'eau. » (Jean Nouvel, Directeur de l'agence Jean Nouvel, http://www.jeannouvel.com/projets/ complexe-aquatique-les-bains-des-docks/).

Dans le quartier de l'Eure se développent un grand nombre d'opérations d'équipements tertiaires, tant privés que publics : Centre commercial des Docks Vauban livré en 2009 pour 69 Millions d'euros, salle de spectacle et de congrès Docks Café terminée en 2016 pour environ 26 Millions d'euros, et plusieurs bâtiments universitaires tels que l'Institut Supérieur d'Études Logistiques, Science PO - INSA et l'Ecole Nationale Supérieure de la Marine.

Du point de vue des espaces publics, la réalisation d'un grand espace vert nommé 'Jardin Fluvial' permet une ouverture des façades du quartier vers les bassins et s'inscrit encore une fois dans une logique de grands noms de l'architecture et de discours sur le port et la mer. La réalisation du 'Jardin Fluvial' est effectuée par phases distinctes entre 2002 et 2006. Elle mobilise des financements provenant du programme européen PIC URBAN et de la Ville du Havre. Le cabinet Obras a été lauréat du Grand prix d'architecture et d'urbanisme pour ce projet de 23 Millions d'euros. En tant qu'espace public, le projet assure la cohérence et le fonctionnement des différentes opérations, notamment des immeubles de logement. En tant qu'élément attractif prépondérant de l'opération urbaine, l'eau joue un rôle éminent dans le discours construit sur la réinterprétation du patrimoine matériel et immatériel de l'espace industrialo-portuaire et du style de vie d'une ville-port (Verdelli et Morucci, 2014). L'eau est mise en visibilité et en résonance dans les choix architecturaux et paysagers 
pour engager l'idée d'une qualité de vie et d'une proximité avec l'environnement maritime (Figure 6).

Figure 6 : Le Jardin Fluvial, une réinterprétation esthétisée de l'environnement portuaire

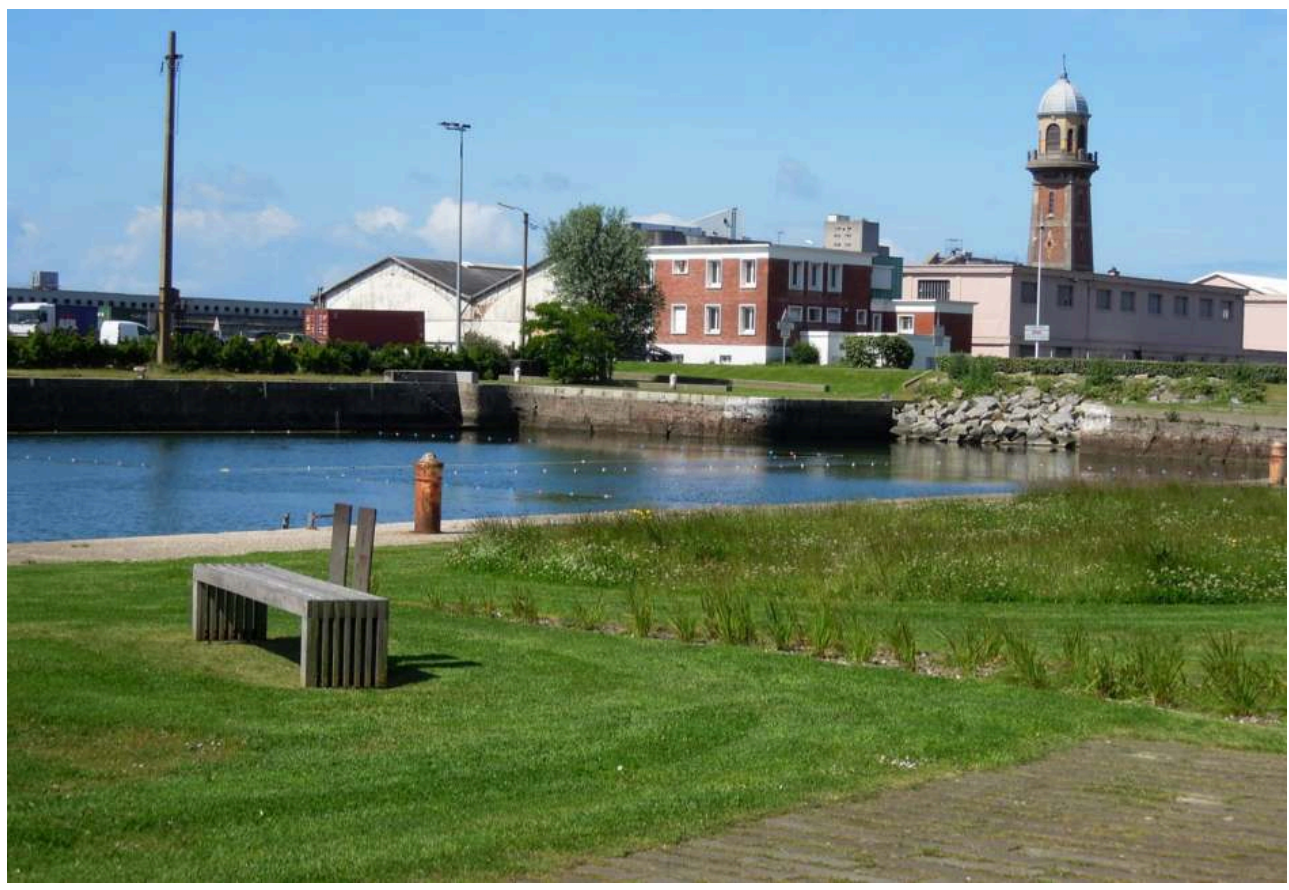

Source : Orillard, 2016

Parmi les nombreuses opérations immobilières (lotissement St Nicolas, opération Bellarmato, programme d'habitat Prony Courbet ...) souvent mixtes entre logements, bureaux, locaux d'activité et/ou commerces, nous remarquons l'exemple des Docks Dombasle, qui sont, jusqu'en 2006 une friche industrielle située sur le quai Nord du bassin fluvial, à la limite sud du quartier de l'Eure. De 2005 à 2009, cette opération mixte de réhabilitation (construction de 25 logements + bureaux + parking) mêle reconversion du bâti ancien et construction neuve de logements hauts de gamme en réinterprétant de manière contemporaine la forme portuaire des anciens docks.

"Through its scale, rhythm shape and materials, the project forms an integral part of a re-envisioned harbour landscape, creating the transition between a domestic scale and the greater harbour landscape." ${ }^{15}$ (Agence Harmonic + Masson \& associés, 'Le Havre, Docks Dombasle, quartier St Nicolas', http://www.hamonic-masson.com/ Le-Havre-Docks-Dombasles-quartier).

Cette opération de 3,2 Millions d'euros est également à l'image de la stratégie de la maîtrise d'ouvrage locale (Ville du Havre) : la délégation de la maitrise d'ouvrage à un cabinet privé, mais surtout - car plus volontariste - le rachat foncier massif des parcelles. On notera également le recours au travail amont d'une équipe d'Assistance à Maîtrise d'Ouvrage (AMO) pour le compte de la Ville du Havre dont le rôle est de mettre en œuvre le pilotage stratégique du projet urbain. Du point de vue des choix architecturaux et paysagers, dès 2001, l'architecte coordinateur de cette AMO (agence Bruno Fortier-Bloch) propose un plan masse comprenant l'organisation du site, son plan de circulation, les cahiers des charges de commercialisation des lots ainsi qu'un cahier des charges de prescriptions paysagères destinés aux opérateurs immobiliers. Toutes les opérations sont communément tournées vers un discours fédérateur. Les 
intentions de cette coordination présentent les bassins historiques du centre-ville comme l'emblème paysager, urbain, écologique et identitaire de la qualité du cadre de vie (Agenda 21, 2013). Au lieu de bassins industriels appréhendés comme des coupures urbaines, des friches polluées ou de hangars désertés, ils procèdent à une "réimagination du centre-ville » (Rousseau, 2008).

29 Analysant Le Havre, Hélène Bailleul (2008) montre que l'image produite valorise les formes d'habitats innovants, un patrimoine industrialo-portuaire réinterprété, un cadre de vie maritime, une offre commerciale variée, une convivialité de quartier voire un 'esprit village'. Cette logique de renversement d'image s'observe dans l'évolution du nom du quartier: le 'quartier de l'Eure', nom associé à un quartier industrialoportuaire en déshérence et enclavé, devient le 'quartier Saint Nicolas', auquel est associé l'image du clocher et du centre-ville. Faisant appel à la valeur ajoutée patrimoniale, le processus de requalification peut être lu comme une nouvelle forme d'appropriation identitaire de l'espace (Veschambre, 2007) dont on peut se demander si in fine, le résultat produirait une gentrification des quartiers industrialo-portuaires.

Dans une seconde partie, nous montrons que le projet urbain de l'Eure ne cherche pas uniquement la réintégration du quartier vers plus de centralité, le remplacement de l'image, la réhabilitation urbaine et paysagère de friches industrielles : il s'assigne aussi un objectif de transformation économique aussi bien que sociale (Bridonneau, 2014). En effet, la production de ces images urbaines construites laisse de côté l'histoire d'un quartier de l'Eure marginalisé (Boquet, 2009) et d'une population précaire qui n'est pas intégrée dans le futur du projet urbain. Au-delà du recours à un imaginaire agréable, la prise en compte des contraintes liées à la présence de l'eau, notamment le risque d'inondation, permet de légitimer la requalification 'par le haut', qui pose sous plusieurs aspects les bases d'une gentrification indirecte des quartiers portuaires.

\section{La prise en compte des risques d'inondation : levier de processus de gentrification indirecte des quartiers portuaires}

31 Jusqu'ici nous avons montré que la valorisation des aspects patrimoniaux et ludiques de l'eau dans le projet urbain de l'Eure constituait une réponse stratégique de la maîtrise d'ouvrage locale (Ville du Havre et Communauté d'Agglomération) au désenclavement des quartiers portuaires pour les réintégrer dans les fonctions du centre-ville. Au-delà, ce projet urbain est un signal d'appel aux populations ayant une propension locale à consommer pour qu'ils réintègrent Le Havre. Ainsi, le transfert d'identité porté par un usage politisé de l'élément 'eau' transforme la requalification 'par le haut' des quartiers portuaires en processus de gentrification indirecte. Dans quelle proportion les mesures de sécurité collective mises en œuvre pour prévenir le risque d'inondation du quartier y participent-elles?

\section{Plus qu'une requalification "par le haut" du quartier de l'Eure : une gentrification indirecte}

À plusieurs égards, la requalification urbaine des quartiers de l'Eure, débutée en 1998, peut être qualifiée de requalification 'par le haut': l'objectif est d'implanter des 
activités dites 'à forte valeur ajoutée' et de renouveler l'offre de logement autour d'une montée en gamme. Le délégué général au développement des quartiers Sud énonce, au milieu du processus de requalification, que :

« Le domaine portuaire environnant les quartiers Sud abrite plusieurs sites (bassins, terre-pleins, quais, bâtiments...) délaissés de façon plus ou moins permanente par l'activité portuaire et industrielle. Ce patrimoine, qui bénéficie d'une position privilégiée au bord de l'eau, constitue une opportunité exceptionnelle pour l'implantation de fonctions urbaines à forte valeur ajoutée (équipements d'agglomération, immobilier d'entreprise, résidence, etc.). ( (Dorbane et al., 2007).

Mais plus qu'une opération de requalification et de mixité sociale 'par le haut', le projet urbain du quartier de l'Eure accumule plusieurs signaux de la mise en œuvre d'un processus de gentrification indirecte.

D'une part, nous parlons de 'processus' car les éléments qui vont être amenés dans le texte sont mis en place depuis le milieu des années 2010. D'autre part, nous insistons sur le terme de gentrification pour plusieurs raisons. D'abord, les pouvoirs publics locaux sont les initiateurs et les pilotes du projet. Les friches portuaires du quartier de l'Eure ont longtemps été considérées comme les 'bas fond' du Havre ${ }^{16}$. Aujourd'hui, il s'agit d'une zone de développement stratégique caractérisée par un investissement fort des pouvoirs publics. Le foncier est vendu par un établissement public (Grand Port Maritime du Havre) à une collectivité locale (Ville du Havre). La maîtrise d'ouvrage se base sur une acquisition prioritaire du foncier, un investissement public dans des infrastructures commerciales, culturelles, de loisirs, d'enseignement supérieur, tout comme dans le cadre urbain et paysager. Ensuite, au-delà d'un objectif de mixité, les statistiques récentes sur le quartier montrent : la diminution du logement social, la hausse des prix des logements (neufs et rénovés), la diminution de la part des catégories sociales de type 'ouvriers' et l'augmentation des professions intellectuelles supérieures.

Les fonctions urbaines à forte valeur ajoutée implantées dans le quartier de l'Eure peuvent être illustrées par la diminution de la part du logement social dans le quartier (31,2\% en 1999 contre $26,8 \%$ en 2013) au profit de la création de logements neufs (AURH, 2018) et de la hausse rapide des prix pratiqués sur le marché du foncier et du locatif. Ainsi, les prix de loyer sur les parties habitées du quartier de l'Eure rejoignent en 5 ans les prix moyens pratiqués sur Le Havre (Figure 7).

Figure 7 : Évolution comparative des prix des loyers par typologie de logement entre le quartier de l'Eure et la ville du Havre : progression des prix après la requalification urbaine de l'Eure

\begin{tabular}{|l|l|l|l|l|l|l|}
\hline \multirow{2}{*}{ Type de logement } & \multicolumn{4}{l}{ Quartier de l'Eure } & \multicolumn{2}{l|}{ Le Havre } \\
\cline { 2 - 7 } & 2006 & 2011 & Évolution & 2006 & 2011 & Évolution \\
\hline T1 & $255 €$ & $344 €$ & $+35 \%$ & $326 €$ & $344 €$ & $+6 \%$ \\
\hline T2 & $340 €$ & $427 €$ & $+26 \%$ & $426 €$ & $463 €$ & $+9 \%$ \\
\hline T3 & Non significatif, effectifs réduits & $565 €$ & & $583 €$ & $589 €$ & $1 \%$ \\
\hline
\end{tabular}

Source : Service de l'Amélioration de l'Habitat, Ville du Havre, 2013 

prix des loyers, ainsi qu'une dynamique de spéculation portée par les investissements privés immobilier et financier depuis les années 2000. Le quartier de l'Eure est présenté comme un quartier dans lequel il faut investir : «Le quartier des Docks est dorénavant très recherché pour le logement: la croissance de la population a été de plus de $5 \%$ entre 1999 et 2009, le prix moyen des loyers ayant dépassé les $10 € / \mathrm{m}^{2} / \mathrm{h} . c h$ " (Courlis conseil, 2014). Pour cela, le travail sur une architecture de qualité, la revalorisation des bassins portuaires et un environnement paysager permettent d'accroître la valeur économique du site :

«Les logements vendus sont assez chers : 2800 - $3000 €$ le mètre carré car ce sont des logements neufs, en front de port, le cadre est agréable avec les jardins, la proximité avec la gare, etc. Ces logements se sont bien vendus. On assiste à une sorte de mixité 'par le haut'. » (Architecte, cabinet Obras, avril 2014)

L'accroissement des prix du foncier et du locatif, l'incitation aux stratégies spéculatives, mais aussi l'instrumentalisation de la valeur esthétique, touristique et ludique de l'eau s'adressant aux imaginaires des actifs des couches supérieures, des étudiants et des retraités sont autant de signaux concrets et symboliques de gentrification indirecte dans les quartiers portuaires du Havre.

plus, deux autres tendances y contribuent. En parallèle de la hausse des prix du foncier et du locatif se produit le départ des catégories précaires et le renouvellement des catégories socio-professionnelles. Depuis les années 1980, les anciens îlots urbains situés plus au Sud-Est du quartier abritent une population qui se précarise socialement et économiquement. En même temps, le parc de logements pose des problèmes de vétusté, d'hygiène et de sécurité et induit un enclavement des occupants.

«L'analyse du profil socio-économique des ménages locataires, de leurs mobilités et des stratégies immobilières des propriétaires bailleurs traduit la consistance d'un parc privé dégradé régi par des logiques d'investissement et de rentabilisation peu enclines au respect de la légalité et des règles de décence. De ce fait, le parc de logements vétustes fait office de 'parc social de fait', où s'accumulent les populations dont la situation économique, familiale, psychologique ou sociale ne leur permet pas d'intégrer le parc social 'classique'. » (Service Amélioration de l'Habitat, 2013).

En 10 ans, cette population précaire se retrouve au milieu d'un chantier de requalification du bâti, entourée d'activités, de symboles, de restaurants qui ne lui sont pas destinés. Nous parlons de gentrification indirecte car si, dans un premier temps, le renouvellement du quartier s'accompagne d'une décroissance de la population du quartier qui passe de 8218 habitants en 1999 à 8021 habitants en 2010, soit une baisse de 2,4\% (Service Amélioration de l'Habitat, 2013), nous observons dans un second temps le retournement du profil des habitants du quartier en termes de catégories socio-professionnelles. L'évolution de la population habitante à l'échelle du quartier se traduit, dans un court laps de temps, par la baisse du nombre d'ouvriers (-5\%), tandis que la présence de la classe moyenne se renforce avec $+2 \%$ d'employés, $+1,8 \%$ de professions intermédiaires et $+0,6 \%$ de cadres ou professions intellectuelles supérieures (Figure 8). Ces chiffres montrent des tendances qui nécessitent d'être confirmées quantitativement. Dans son bilan récent des opérations de renouvellement, l'Agence d'Urbanisme de la Région du Havre et de l'Estuaire de la Seine (AURH) signale sans détour que : «l'implantation de plusieurs centaines de logements neufs, en grande partie privés, ont permis d'accueillir une nouvelle population de classes moyennes 
( $35 \%$ de cadres supérieurs et professions intermédiaires contre $19 \%$ il y a 5 ans) » (AURH, 2018). Ce à quoi le rapport ajoute que la proportion de personnes diplômées de l'enseignement supérieur a doublé depuis 2008 atteignant $25 \%$.

Figure 8 : Renouvellement de la population du quartier de l'Eure entre 1999 et 2010

\begin{tabular}{|l|l|l|l|}
\hline \multirow{2}{*}{ Pop 15 ans ou plus dont : } & 1999 & 2010 & \multirow{2}{*}{ Évolution 1999/2010 } \\
\cline { 2 - 4 } & 6352 & 6128 & \\
\hline Agriculteurs exploitants & $0,0 \%$ & $0,1 \%$ & $0,1 \%$ \\
\hline Artisans, Commerçants, Chefs entreprise & $2,0 \%$ & $1,8 \%$ & $-0,3 \%$ \\
\hline Cadres, Professions intellectuelles supérieures & $1,6 \%$ & $2,2 \%$ & $0,6 \%$ \\
\hline Professions Intermédiaires & $6,9 \%$ & $8,8 \%$ & $1,8 \%$ \\
\hline Employés & $19,7 \%$ & $21,7 \%$ & $2,0 \%$ \\
\hline Ouvriers & $28,1 \%$ & $23,0 \%$ & $-5,0 \%$ \\
\hline Retraités & $14,1 \%$ & $16,1 \%$ & $2,0 \%$ \\
\hline Autres & $27,5 \%$ & $26,4 \%$ & $-1,2 \%$ \\
\hline
\end{tabular}

Source : Service de l'Amélioration de l'Habitat, Ville du Havre, 2013

41 Ainsi, la hausse du prix du foncier et du locatif, le remplacement des activités industrielles et ouvrières par des lieux dédiés aux populations aisées, le départ des ouvriers et l'évolution des profils sociodémographiques combinés à la transformation symbolique de l'espace urbain par des politiques de protection du patrimoine de la part des pouvoirs publics (Semmoud, 2005) engendrent, directement ou indirectement, la gentrification de la population résidente. Au Havre, « la réinvention des espaces urbains passe par des interventions 'physiques', mais joue avant tout sur la symbolique de l'espace » (Bailleul, 2008). En cela, on peut parler de gentrification car il y a une volonté publique d'opérer un 'basculement social', c'est-à-dire le remplacement des classes populaires et leur relégation hors du grand périmètre du centre-ville (Clerval, 2009), et de créer une rupture dans le processus d'urbanisation précédent pour explorer de nouvelles identités (Smith, Butler, 2007). En 10 ans, le processus du projet urbain du quartier de l'Eure a transformé la fonction du quartier (passage d'une fonction industrielle portuaire à un quartier urbain d'habitat et de services), le paysage (traitement paysager et végétalisé de l'espace portuaire, évolution de la morphologie urbaine via les réinterprétations contemporaines du patrimoine et les constructions neuves), et aussi son peuplement en incitant au remplacement des populations locales précaires.

Plus précisément, nous pouvons dire que la maîtrise d'ouvrage locale met en œuvre dans le quartier de l'Eure une politique de requalification qui amène une gentrification indirecte, terme qui permet de «s'assurer que la pureté conceptuelle de gentrification n'est pas diluée $»^{17}$ comme soulignent en partie ironiquement Smith et Butler (2007). Les 
théories de new build gentrification (Davidson et Lees, 2005) expliquent que la dynamique de constructions neuves dans d'anciennes friches, afin de réintégrer ces espaces dans les centres urbains, se caractérise par un réinvestissement de capital dans les zones délaissées, par une modification de l'environnement construit, du paysage urbain et même des catégories sociales (Rérat et al., 2008).

«Le processus d'éviction n'est dans ce cas pas direct - les zones en question étant inhabitées - mais indirect: la mise en valeur d'un site augmente l'attractivité des quartiers environnants où un phénomène de remplacement de population pourrait se produire à terme. " (Rérat et al., 2008). Au Havre, cette stratégie de gentrification indirecte s'exprime lors de la rénovation du parc bâti existant de la zone Sud-Est du quartier de l'Eure.

Si des contestations locales se matérialisent souvent autour des processus de gentrification, ici il semble que la requalification se déroule 'à bas bruit'. Notre hypothèse réside dans le fait que les hausses de loyer, les formes architecturales et paysagères permettant le changement d'image sont justifiées par la prise en compte des risques d'inondation dans le quartier.

\section{La prise en compte du risque d'inondation, facteur de justification de la gentrification dans le quartier de l'Eure}

Dans le cadre d'une lecture non-naturaliste et davantage géopolitique de la prévention des risques (Pigeon, 2015), nous étudions la possibilité que la prise en compte des règles de construction en zone inondable joue un rôle disculpant dans la montée en gamme des prix du quartier, dans le changement d'image et in fine dans le remplacement de la population.

Dans le quartier de l'Eure, le risque est multicausal (submersion, débordement, ruissellement, saturation des nappes et/ou des réseaux) (Figure 2). La multicausalité rend le risque majeur d'inondation à la fois peu probable ${ }^{18}$ et potentiellement catastrophique. Ce cas précis explique une absence de réglementation spécifique concernant l'inondation dans les quartiers centraux du Havre et sur la partie portuaire. Malgré l'absence de Plan de Prévention des Risques d'Inondation pour réglementer le développement de l'interface ville-port, les contraintes techniques aux opérations urbaines sont issues de deux sources éditées par la maîtrise d'ouvrage elle-même.

La première source peut être qualifié de 'droit souple'. Elle découle des préconisations du cahier de prescriptions architecturales et paysagères, élaboré par l'agence FortierBloch en qualité d'Assistance à Maîtrise d'Ouvrage pour le compte de la Ville du Havre. Ces préconisations permettent d'obtenir un ensemble urbain cohérent malgré la disparité des maîtres d'œuvre dans les diverses opérations.

La deuxième source s'applique réglementairement à l'échelle du territoire communal par le biais du Plan Local d'Urbanisme (PLU). Elle concerne principalement l'application de certaines normes liées à la gestion des réseaux d'eau par le biais des annexes sanitaires ${ }^{19}$. Les deux grandes catégories de contraintes imposées à la conception et à la construction du quartier sont la surélévation et l'interdiction de présence dans les sous-sols.

« Nous on demande :

que le niveau du sol des logements soit à 4 mètre cote NGF

- que les sous sols/parkings ne soient pas reliés au sol gravitaire 
- que toutes les conduites dans les sous sols soient des conduites verrouillées et étanches et résistent à la pression pour gérer la montée des eaux. »

(Direction Cycle de l'Eau, Communauté d'Agglomération de la région Havraise CODAH, juillet 2014)

Ce système de contraintes empêche le stationnement semi-enterré en zone d'affleurement de nappe (Figures 9 et 10). Cette obligation s'avère financièrement très lourde, principalement à cause du cuvelage devant être réalisé pour les parkings, sachant que le nombre de ceux-ci ne peut pas être réduit en raison des préconisations du PLU qui imposent un nombre de places de stationnement par logement. De plus, cette contrainte peut être oubliée par les architectes, ce qui entraîne un retard sur les travaux en cas de refus du permis de construire par la Ville.

«Les architectes regardent souvent les réglementations, mais pas les annexes sanitaires. Très peu consultent en amont pour être conseillés. C'est donc au moment de l'instruction du permis de construire que l'avis peut être négatif car les logements sont considérés comme inondables, alors que le projet est déjà bien avancé et que c'est donc plus difficile. " (Direction Cycle de l'Eau, Communauté d'Agglomération de la région Havraise CODAH, juillet 2014)

Figure 9 : Exemple d'une installation non conforme pouvant entraîner un blocage de permis de construire - Préconisations du PLU relatives au risque d'inondation

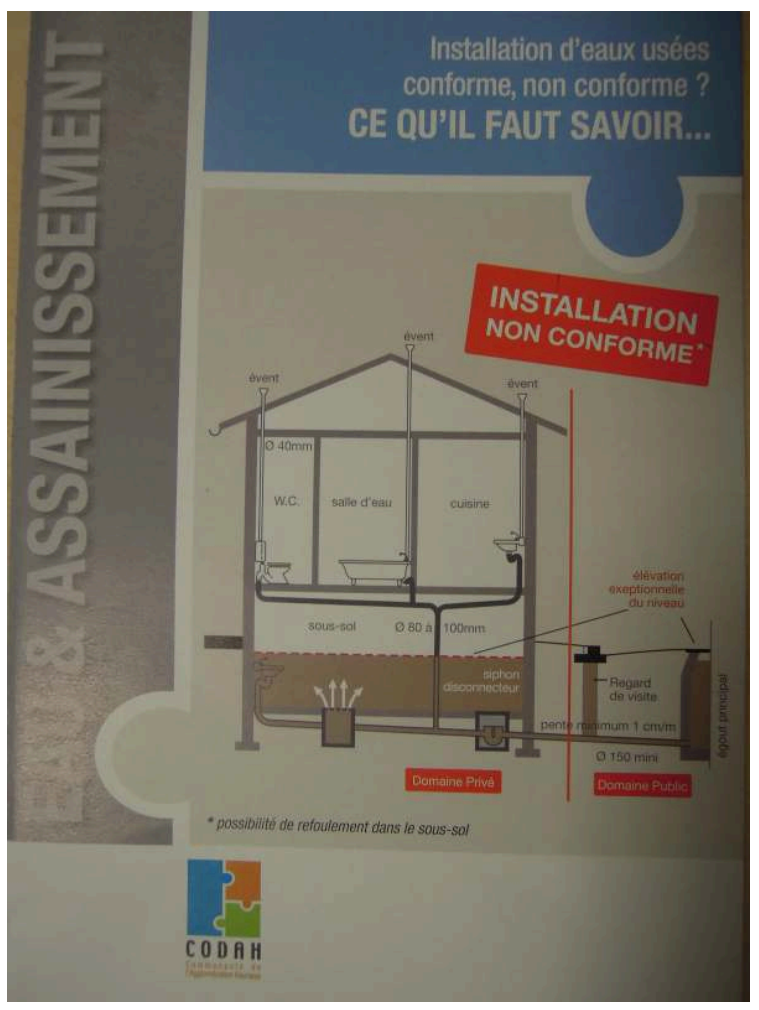

Source : Service Eau et Assainissement, 2014 
Figure 10 : Exemple d'une installation conforme permettant d'éviter le risque d'inondation par remontée d'eau dans les réseaux (inversion gravitaire) - Préconisations du PLU relatives au risque d'inondation

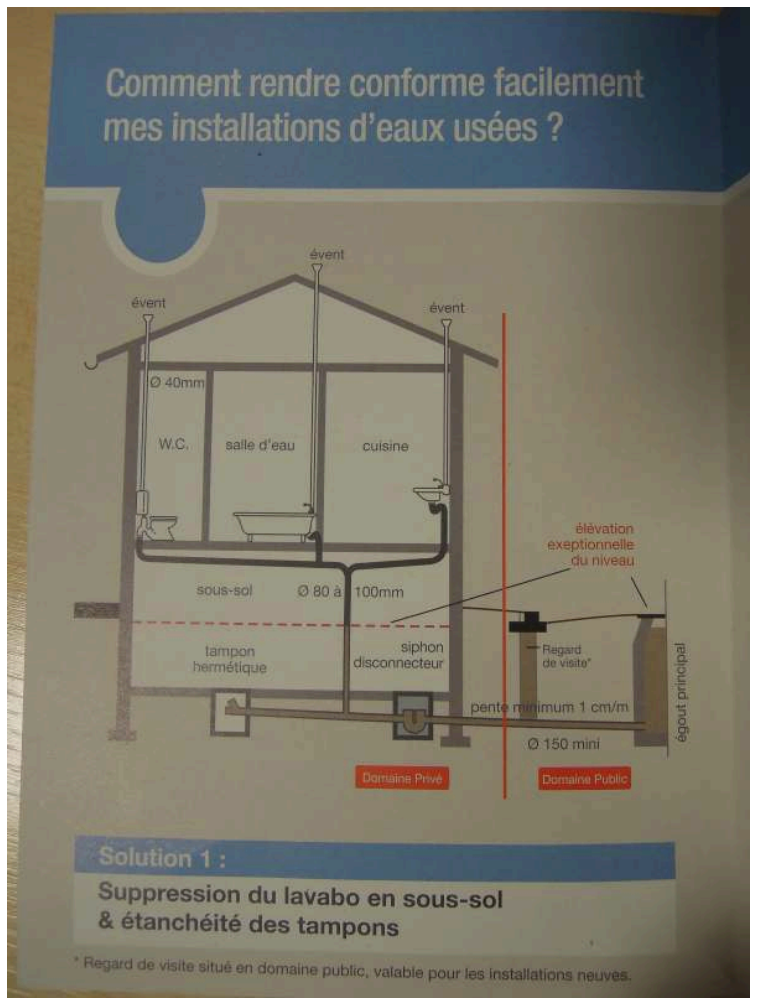

Source : Service Eau et Assainissement, 2014

50 Il existe une complexité ultérieure qui consiste à faire en sorte de concilier (i) la volonté du cahier des prescriptions architecturales et paysagères de ne pas trop monter en verticalité (le quartier ne comporte pas trop d'immeubles de grande hauteur) et (ii) les obligations de surélévation et de non-exploitation des sous-sols imposées par les annexes sanitaires du Plan Local d'Urbanisme.

« La première de ces suggestions, s'agissant de volumes relativement bas $(R+3, R+5)$, conduit à notre sens à privilégier un formalisme en longueur plutôt que vertical. Les jeux d'horizontal devront être affirmés pour créer sur ce site un effet général d'allègement et de liberté. " (Fortier, Boubet, Cahier des prescriptions architecturales et paysagères, $2008: 6$ )

«Tout le monde a opté pour la solution de relever les rez-de-chaussée. C'est quasiment impératif. Ça n'a pas donné lieu à une concertation parce qu'il n'y a pas mille et une façons de résoudre [les contraintes sur l'inondation]. Il faut effectivement construire les rez-de-chaussée plus haut, et construire après selon les cotes de terrain qui n'étaient pas tout à fait identiques. On a essayé d'harmoniser les hauteurs mais c'était lié de toute façon aux cotes des plus hautes eaux. " (Architecte, Atelier Furet, avril 2014)

51 Le respect des différentes contraintes liées à la prévention des risques d'inondation entraîne des surcoûts budgétaires inévitables, car les méthodes de construction standard ne sont pas reproductibles et les temps de réalisation sont augmentés. Pour rentabiliser l'intégration de ces contraintes, le choix proposé par les maîtres d'œuvre, et accepté par la maîtrise d'ouvrage, s'oriente unilatéralement vers un report des coûts sur le prix du foncier et du locatif. En acceptant l'augmentation des loyers et des prix de vente, le projet ne s'adresse pas aux populations locales précarisées. Sous prétexte 
de rentabiliser une opération urbaine et immobilière complexe, l'inondation participe à la justification d'une requalification urbaine qui s'adresse à une population de classe moyenne voire aisée par l'installation de logements de standing.

«Au niveau architectural, ce sont des projets plutôt qualitatifs par rapport à certains projets qu'on voit plus basiques (...) C'est vrai que c'est un secteur qui est pas mal : y'a les bassins...» (Service urbanisme, Ville du Havre, juin 2014).

Au vu des contraintes énoncés dans les annexes sanitaires du Plan Local d'Urbanisme et des prescriptions du cahier architectural et paysager, la complexité de la conception et de la construction liée au caractère inondable de l'espace de projet induit des surcoûts qui se répercutent dans les prix du marché du foncier et qui sont portés par le changement d'image. Pour assumer économiquement le coût important du réaménagement des quartiers portuaires inondables et pour attirer une population ayant une plus forte propension à consommer que celle présente historiquement, la Ville du Havre produit une politique urbaine de gentrification indirecte et tente de prolonger le processus vers les quartiers Sud du Havre par effet boule de neige, vers les quartiers Sud situés plus à l'Est ${ }^{20}$.

\section{La prise en compte du risque d'inondation produit-elle un urbanisme sélectif?}

Plusieurs travaux récents tentent de décrypter les effets induits dans les équilibres de jeux d'acteurs des politiques de renouvellement urbain en zone inondable montrant qu'il s'agit d'espaces de transaction sociale entre acteurs publics et privés (Bonnefond et al. 2017) ou de recomposition des métiers de l'aménagement (Guevara et Gralepois, 2015). Ici, nous souhaitons insister sur le rôle de l'intégration des contraintes liées aux risques d'inondation pour expliquer voire disculper, sous couvert d'arguments de sécurité, une opération urbaine qui gentrifie un îlot d'habitat précaire.

Pour de nombreuses villes françaises, les espaces inondables constituent des espaces stratégiques de développement (Rode, Gralepois, 2017) du fait de leur localisation centrale, de la relative mutabilité du foncier, de la superficie potentiellement libérés ou encore des prix d'acquisition très abordables pour des espaces stratégiques (Orillard, 2014). Autant de qualités qui animent les velléités de développement des politiques d'aménagement et de développement. Mais le caractère inondable des espaces ajoute des contraintes à des projets urbains déjà fort complexes. Lorsqu'une réglementation vient imposer des hauteurs de construction, des circulations d'eau, des mesures de relèvement de l'électricité ou des interdictions d'occupation au sol et en sous-sol, c'est alors autant de coûts induits pour les maitres d'œuvre. Parmi les possibilités qui s'offrent à la maîtrise d'ouvrage locale, il existe soit une prise en charge politique et économique du projet, soit le report des coûts induits sur les caractéristiques de la programmation urbaine.

Dans le second cas, pour les raisons évoquées précédemment, la maîtrise d'ouvrage assume la responsabilité des conséquences politiques, économiques et sociales de ses choix. En tant que contrainte liée au site, l'inondation participe à la justification du besoin de rentabiliser une opération complexe, notamment par la sélection d'une population nouvelle et plus aisée. Alors que la gentrification - de type new build gentrification - est présentée comme le résultat d'un ensemble d'obligations et de contraintes, elle constitue au Havre l'objectif d'une stratégie d'accélération de la 
transformation souhaitée par la maîtrise d'ouvrage locale (Ville du Havre et Agglomération du Havre) des quartiers populaires périphériques au Sud. Dit autrement, dans le cas des villes-ports, et particulièrement du Havre, la gentrification des espaces d'habitats populaires liée à la requalification urbaine du quartier de l'Eure s'appuie sur le respect de la réglementation liée aux risques d'inondation des sites portuaires.

Là où la littérature sur la vulnérabilité urbaine s'inquiète souvent de potentiels cumuls de risques entre des expositions spatiales aux dangers et des vulnérabilités sociales (occupation informelle, population précarisée, infrastructure fragile...), nous constatons l'effet inverse au Havre, et nous faisons l'hypothèse que cette option prise par les maitrises locales d'ouvrage de reporter le coût des opérations en zone inondable sur des opérations immobilières haut de gamme se produira dans les projets urbains en zone inondable en cours de conception que nous avons eu l'occasion d'observer (Pirmil - Les Isles à Rezée ${ }^{21}$ ou Seine-Ardoines aux Ardoines ${ }^{22}$ ). Les quartiers dits 'résilients' qui intègrent la prise en compte des risques naturels, produisent des quartiers d'habitat à forte valeur ajoutée, dédiés à des habitants possédant un capital culturel et économique élevé. Les projets urbains cherchent à valoriser le patrimoine des espaces en requalification, mais sans acquérir ni la mémoire du lieu, ni la compréhension des potentiels dangers d'inondation. Ainsi, l'intégration des contraintes liées à la prévention des risques et la nécessité de rentabiliser des opérations rendues complexes font émerger un urbanisme certes conscient de l'inondabilité des sites mais également sélectif dans la population attendue sur ces opérations urbaines.

Sachant qu'une des caractéristiques du site portuaire est d'être une zone inondable, peut-on dire que les contraintes liées à l'inondation permettent de rendre acceptable voire obligatoire - la gentrification induite? Les études citées jusqu'alors sur la gentrification soulignent l'importance du caractère progressif du processus pour une bonne acceptation sociale. Comme l'énoncent Blanck et Siou (2009), la gentrification est susceptible de générer cette contestation. L'absence de contestations citoyennes à l'encontre du projet urbain impulsé par les pouvoirs publics pose question. D'où vient l'acceptabilité sociale d'un projet urbain rapide et radical sur un quartier ayant une longue tradition de luttes sociales et syndicales (Barzman, 2012) ?

Le renouvellement du quartier de l'Eure, qui débute en 1998, produit en 10 ans la gentrification des quartiers de friches portuaires et ne génère pourtant pas de contestation. Quelques échanges sur des forums de blog Internet constituent des témoignages d'une certaine nostalgie de l'ambiance portuaire :

«Le processus de désindustrialisation de la ville du Havre se poursuit. Dans deux à trois ans, de nouveaux habitants vont s'approprier ces espaces réaménagés. Beaucoup ignoreront que, pendant des décennies, voire un siècle et demi, ce quartier a été le centre des embarquements des marins, de l'embauche des dockers, des allées et venues du millier d'ouvriers de Caillard employés à la construction des grues portuaires et à la réparation des navires. » (Bastide, 2006)

Les institutions en charge de la requalification urbaine (Ville du Havre, agence d'urbanisme de la région havraise - AURH - et Agence nationale pour la rénovation urbaine - ANRU - par exemple) ont également relevé la dimension nostalgique des discours des habitants locaux :

«On peut citer également la nostalgie de certains habitants du quartier de l'Eure qui craignent de voir leur quartier perdre son identité, devenir 'trop lisse', ceci en lien avec la construction des ensembles immobiliers du secteur Saint Nicolas. » (AURH, point d'étape de la convention ANRU, 2012) 

l'insertion du projet urbain dans un tissu portuaire comportant d'importantes surfaces de friches industrielles, non accessibles au public depuis les années $1970^{23}$, induit une faible connaissance ou un faible attachement au quartier de l'Eure, c'est-à-dire un imaginaire collectif partiellement effacé et donc plus facilement 'ré-interprétable' par les politiques urbaines.

63 Ainsi, la prévention des risques participe à justifier un appel symbolique fort à destination d'une population aisée. Le système de contrainte réglementaire lié à l'inondation contribue indirectement à renouveler 'vers le haut', d'une part, l'offre de logement en justifiant une hausse du prix des terrains et des loyers censés compenser les contraintes de construction et, d'autre part, à renouveler le style architectural contribuant au changement d'image du quartier qui passe d'une image de quartier populaire à celle d'un quartier 'branché'.

\section{Conclusion}

$\mathrm{Au}$ terme de cet article, nous retenons que la gentrification indirecte constitue un effet induit des opérations de requalification urbaine des espaces industrialo-portuaires. Pour cela, l'article s'attache à montrer comment les contraintes liées à la prévention des inondations présentent une opportunité réglementaire pour poursuivre un objectif de gentrification en rendant nécessaire le renouvellement d'un habitat précaire par du logement de haut standing.

Dans le contexte concurrentiel des villes françaises, les villes-ports ayant historiquement axé leur développement vers une économie secondaire, se tournent depuis les années 1990 vers une économie résidentielle et présentielle. Les stratégies urbaines des décideurs publics visent à transformer l'image des espaces industrialoportuaires pour en faire des lieux attractifs pour de nouveaux résidents ayant une forte propension à consommer. La politique publique de requalification génère une gentrification, c'est-à-dire, dans les quartiers portuaires du Havre, un renouvellement social via une stratégie de renouvellement urbain. Dans le cas de quartiers portuaires français, la reconquête des délaissés portuaires ne se fait ainsi pas sous la forme d'une gentrification progressive via différentes vagues d'évolution sociale, mais plutôt sous l'impulsion d'une gentrification planifiée résultant d'une stratégie urbaine visant à attirer les populations ayant de fortes propensions à consommer. Initiée par une politique urbaine, portée par des investissements publics dans des équipements emblématiques, répercutée dans une logique spéculative par l'investissement privé, la 
transformation du tissu urbain du quartier de l'Eure se propage aujourd'hui vers les quartiers portuaires encore plus au Sud.

Alors qu'à ses débuts (selon la définition de Ruth Glass de 1964) la gentrification s'apparentait à une démarche de réappropriation spontanée des quartiers populaires par une population possédant un capital culturel et économique plus élevé, elle est aujourd'hui «souvent la résultante d'incitations municipales explicites par des politiques publiques de réhabilitation de l'habitat et des quartiers, qui non seulement améliorent les conditions résidentielles mais accroissent l'attractivité par l'image de la transformation projetée.» (Introduction, Sociétés contemporaines, 2006). Considérée par de nombreuses autorités locales "comme une politique de développement économique local et comme une solution au déclin urbain " (Bidou-Zachariasen, Authier, 2003), la gentrification - ou a minima l'acceptation passive d'une gentrification indirecte - devient un des acquis de la politique publique volontariste de requalification urbaine dans les quartiers portuaires urbains. Dans le cas des quartiers de l'Eure au Havre, il ne s'agit pas uniquement d'une stratégie de mixité sociale, mais bien d'une transformation - disons même un remplacement - à la fois de l'image, de l'histoire, du patrimoine, des activités économiques, des fonctions urbaines et aussi de la population.

\section{BIBLIOGRAPHIE}

ADAM M. (2016), La production de l'urbain durable. L'enrôlement des concepteurs et des habitants par l'intégration des contradictions, Thèse de Doctorat, soutenance le 14 juin 2016, Université François-Rabelais de Tours, 2135p. http://www.applis.univ-tours.fr/theses/2016/ matthieu.adam_4599.pdf consulté le 31/05/2018.

AGENCE D'URBANISME DE LA RÉGION DU HAVRE ET DE L'ESTUAIRE DE LA SEINE (2012), Seine Gateway 1.0. Préfiguration du Gateway de la Seine et éléments clefs de mise en œuvre, 64p.

AGENCE D'URBANISME DE LA RÉGION DU HAVRE ET DE L'ESTUAIRE DE LA SEINE (2018), Quartiers sud du Havre : réinventer l'interface ville-port, Rapport Retour sur 20 ans d'intervention, Observatoire habitat et foncier, 28p.

http://www.aurh.fr/media/aurh_obs_hab_foncier_qs_e_1502__020462000_1413_24042018.pdf, consulté le 31/05/2018.

AGENCE D'URBANISME DE LA RÉGION DU HAVRE (2012), Agence Nationale de la Rénovation Urbaine : Quartiers Sud du Havre, Point d'étape de la convention, étude n 1433, 78p. http:// www.aurh.fr/media/

aurh_anruquartierssudpointetapeconvention_e1433__045911200_1536_24042014.pdf consulté le $31 / 05 / 2018$.

AGENCE D’URBANISME DE LA RÉGION HAVRAISE ET DE L'ESTUAIRE DE LA SEINE (2016), Communauté de l'agglomération havraise - CODAH. Les données clés de l'Estuaire de la Seine, $12 \mathrm{p}$.

http://donneescles.aurh.fr/pub/downloads/247600596.pdf, consulté le 31/05/2018 
AGENDA 21 (2013), Le Havre, ville durable : enjeux et objectifs, 2013.

BACHIMON P. (2014) « Paradoxales friches urbaines », L’Information géographique, 2014/2, pp. 42-61.

BAILLEUL H. (2008), « Les nouvelles formes de la communication autour des projets urbains : modalités, impacts, enjeux pour un débat participatif », Métropoles, 2008/3, pp. 98-139. http:// www.ramau.archi.fr/IMG/pdf/communications-projets-urbains.pdf , consulté le 31/05/2018

BARZMAN J. (2012), « Conflits et négociations au Havre avant et après les grandes réformes portuaires ", L'Espace Politique, 2012/1, 16p. https://journals.openedition.org/espacepolitique/ 2242 , consulté le $31 / 05 / 2018$

BASTIDE M. (2006), « Sauver la mémoire du quartier industriel Saint Nicolas de l'Eure au Havre », Revue Départementale d'Histoire Sociale, Institut CGT d'Histoire sociale de Seine Maritime, $\mathrm{n}^{\circ} 26$, 11p. http://gilles.pichavant.pagesperso-orange.fr/ihscgt76/num26/num26page2.htm, consulté le $31 / 05 / 2018$

BENKO G. (1999), « Stratégies de communication et marketing urbain », Pouvoirs locaux, $\mathrm{n}^{\circ} 42$, III, pp. 12-18.

BIDOU-ZACHARIASEN C., AUTHIER J-Y. (2008) « La question de la gentrification urbaine », Espaces et sociétés, 2008/1, pp. 13-21.

BLANCK J., SIOU H. (2009), La gentrification : un phénomène urbain complexe et son utilisation par les pouvoirs publics, Note de lecture, Master Stratégies Territoriales et Urbaines, Sciences Po Paris.

BONNEFOND M., FOURNIER M., SERVAIN S., GRALEPOIS M. (2017) « La transaction foncière comme mode de régulation en matière de protection contre les inondations. Analyse à partir de deux zones d'expansions de crue : l'île Saint Aubin (Angers) et le déversoir de la Bouillie (Blois) », Risques Urbains, 2017/ 1, 17p. https://www.openscience.fr/IMG/pdf/iste_uris17v2n6.pdf consulté le 31/05/2018.

BOQUET M. (2009) « Ségrégation et transformation urbaine : quelle évolution de l'espace havrais?», M@ppemonde, 2009/3, 15p.https://mappemonde-archive.mgm.fr/num23/articles/ art09304.html consulté le 31/05/2018.

BOURDIN A. (2001) « Comment on fait la ville, aujourd'hui, en France », Espaces et sociétés n 105, pp. 147-166.

BRIDONNEAU M. (2014) « Déplacer au nom de la sauvegarde patrimoniale et du développement économique ? Analyse multiscalaire du programme de resettlement à Lalibela (Éthiopie) ", L'Espace Politique, $\mathrm{n}^{\circ} 22$, 22p. https://journals.openedition.org/espacepolitique/2941 consulté le $31 / 05 / 2018$

BROWN P. H. (2009), America's waterfront revival: Port authorities and urban redevelopment. The city in the twenty-first century, Philadelphia: University of Pennsylvania Press, 240p.

CAUCHI-DUVAL N., BEAL V. et ROUSSEAU M. (2016) « La décroissance urbaine en France : des villes sans politique », Espace populations sociétés, 2016/ 1, 29p. https:// journals.openedition.org/eps/6112 consulté le 31/05/2018

CHESNEL H. (2015) « Des enjeux différenciés en matière de santé dans les quartiers des grandes agglomérations », INSEE Analyses Pays de la Loire, n 23, 4p. https://www.insee.fr/fr/ statistiques/1304129 consulté le 31/05/2018. 
CLERVAL A. (2005) « David BROOKS, 2000, Les Bobos, Les bourgeois bohèmes, trad. Par THIRIOUX M. NABET A., Paris, Le livre de poche, 314p. », Cybergeo, 9p. http://journals.openedition.org/ cybergeo/766_consulté le 31/05/2018.

CLERVAL A. (2009) « Les politiques publiques face à la gentrification. Le cas de Paris intra-muros » in Vallat C., Delpirou A., Maccaglia F., Pérennité urbaine ou la ville par-delà ses métamorphoses, Paris, L'Harmattan, pp. 139-151.

COCHARD N. (2013) «Les bas-fonds d'une ville portuaire : l'exemple du Havre au prisme de la presse et de la littérature locales ", Médias 19, numéro " Enquêtes dans les bas-fonds et le monde criminel », 20p. http://www.medias19.org/index.php?Id=13395, consulté le 1/06/2018.

COURLIS CONSEIL (2014), Faire revivre l'architecture traditionnelle havraise : concevoir l'habitat contemporain. Conseil en investissement immobilier et financier.

CUSIN F. (2008) «Économie résidentielle et dynamiques sociologiques de l'attractivité des territoires », revue Pour, n 199, pp. 69-80. https://www.cairn.info/revue-pour-2008-4page-69.htm consulté le 31/05/2018.

DAVEZIES L. (2009) « L'économie locale 'résidentielle'« , Géographie, économie, société, 2009/1, pp. 47-53. https://www.cairn.info/revue-geographie-economie-societe-2009-1-page-47.htm consulté le 01/06/2018.

DAVIDSON M., LEES L. (2005) « New-build 'gentrification' and London's riverside renaissance », Environment and Planning A, vol. 37, pp. 1165-1190. http://wordpress.clarku.edu/mdavidson/ files/2011/04/Davidson-and-Lees-2005-New-Build-Gentrification.pdf consulté le 01/06/2018.

DESFOR G., LAIDLEY J., STEVENS Q., \& SCHUBERT D. (2012), Transforming urban waterfronts: Fixity and flow, Oxon: Routledge, 353p.

DIRECTION RÉGIONALE À L'ÉNERGIE, L'AMÉNAGEMENT ET AU LOGEMENT (2016), Territoire à Risque d'Inondation, Cartes de surfaces inondables du Havre

http://www.normandie.developpement-durable.gouv.fr/cartes-des-surfaces-inondablesa663.html, consulté le 01/06/2018

DORBANE O., CHEDOT C., DAVOULT D. (2007), "Plan the City with the Port", PCP Project, Newsletter $\mathrm{n}^{\circ}$ 7, 11p. www.rop.lv/ru/smi/zagruzki/doc_download/13-pcp-newsletter-7.html consulté le 01/06/2018.

ENTREPRISES TERRITOIRES ET DÉVELOPPEMENT (2005), Les notes de l'observatoire : l'attractivité territoriale dans les projets d'agglomération et de pays. http://fr.calameo.com/read/ 004299699924dcd36af05 consulté le 01/06/2018.

GALLARD-DAUJAN S. (2012), La mixité sociale au risque de la gentrification : Classes moyennes et cohésion sociale, l'exemple du quartier de la Goutte D'Or, Mémoire de fin d'étude de l'ITSRS, Diplôme d'État d'ingénierie sociale, 137p. http://www.cedias.org/index.php? lvl=notice_display\&id=78148 consulté le 01/06/2018.

GRALEPOIS M., RODE. (2017) «L'urbanisme résilient déforme-t-il la ville ? », Risques Urbains, 2017/1, 18p. https://www.openscience.fr/IMG/pdf/iste_uris17v2n1.pdf consulté le 01/06/2018.

GRAS P. (2010), Le temps des ports : déclin et renaissance des villes portuaires (1940-2010), Paris : Tallandier, 298p.

GRAVARI-BARBAS M. (2004) « Politiques patrimoniales locales : quelles marges de manœuvre pour une « gouvernance patrimoniale »? Les cas d'Angers et du Havre », Pouvoirs locaux, $n^{\circ} 63$, pp. 84-93. 
GUEVARA S., GRALEPOIS M. (2015) «L'adaptation aux risques d'inondation façonnée par les métiers de la ville ", Développement durable et territoires, 2015/3, 20p. https:// journals.openedition.org/developpementdurable/11014 consulté le 01/06/2018.

HAROPA (2017), Etudes statistiques du Port du Havre, site officiel du regroupement des trois Grands Ports Maritimes du Havre, Rouen et Paris, 1p. http://www.haropaports.com, consulté le 01/06/2018.

HEIN C. (2011), Port Cities: Dynamic Landscapes and Global Networks, Abingdon, Oxon \& New York: Routledge, 304p.

INGALLINA P. (2001), Le projet urbain, Paris, Presses Universitaires de France, 127p.

INSTITUT NATIONAL DE LA STATISTIQUE ET DES ÉTUDES ÉCONOMIQUES (2014), Emplois maritimes et recul des emplois industriels dans les ports du Havre et de Rouen, Statistiques. https://www.insee.fr/fr/statistiques/2528900, consulté le 1/06/2018

INSTITUT NATIONAL DE LA STATISTIQUE ET DES ÉTUDES ÉCONOMIQUES (2015), L'emploi portuaire, Statistiques, https://www.insee.fr/fr/statistiques/3301091, consulté le 1/06/2018

INSTITUT NATIONAL DE LA STATISTIQUE ET DES ÉTUDES ÉCONOMIQUES (2006), Analyse de la zone d'emploi au Havre.

JOURDAN S. (2008) « Un cas aporétique de gentrification : la ville de Marseille, Méditerranée », Revue de Géographie des Pays Méditerranéens, $n^{\circ}$ 111, pp. 85-90. https:// journals.openedition.org/mediterranee/2788 consulté le 31/05/2018.

LE BART C. (2011), “Marketing territorial”, in Pasquier R., Guigner S., Cole Al., Dictionnaire des politiques territoriales, Paris, Presses de Sciences Po, pp. 318-346.

LOUNGOU S. (2014) « La destruction des villages de pêcheurs au sud de Libreville. Une opération entre impératif sécuritaire et spéculation foncière ", L’Espace Politique 2014/ 1, 19p. https:// journals.openedition.org/espacepolitique/2997 consulté le 31/05/2018.

LUSSAULT M. (2013), Le Millenium Park, Chicago, Tous Urbains, $n^{\circ}$ 3, $64 \mathrm{p}$.

NINNIN J. (2014), « Le rêve carioca : entre planification urbaine et déplacements forcés de population », L'Espace Politique, ${ }^{\circ}$ 22, 19p. https://journals.openedition.org/espacepolitique/ 2950 consulté le 01/06/2018.

NOVARINA G. (2003), Plan et projet l'urbanisme en France et en Italie, Paris, Anthropos, 223p.

ORILLARD F. (2014), Etude de la contrainte d'inondation dans les espaces urbains. Cas du quartier de l'Eure au Havre, Stage de fin d'études, École Polytechnique Universitaire, Université de Tours, réalisé à l'Office Régional des Risques Majeurs de l'Estuaire de la Seine.

PIGEON P. (2015), « Risque digue : une justification à la relecture systémique et géopolitique des risques environnementaux ", L'Espace Politique, n² 24, 25p. https://journals.openedition.org/ espacepolitique/3256 consulté le 01/06/2018.

PORTER M. (2003), L'avantage concurrentiel. Comment devancer ses concurrents et maintenir son avance, Paris, Dunod, 656p.

PRELORENZO C. (2010) « Le retour de la ville portuaire », Cahiers de la Méditerranée, vol. 80, p. 157-167. https://journals.openedition.org/cdlm/5239 consulté le 01/06/2018.

RAPPORT DU SENAT (2014), l'évolution des finances locales à l'horizon 2017, Mission d'information Délégation aux Collectivités Territoriales, Dallier P., Guené C., Mézard J., rapport n 95, 126p. https://www.senat.fr/rap/r14-095/r14-0951.pdf consulté le 01/06/2018. 
REGHEZZA-ZITT M. (2015) « Territorialiser ou ne pas territorialiser le risque et l'incertitude. La gestion territorialisée à l'épreuve du risque d'inondation en Île-de-France ». L'Espace Politique, vol. 2, n 26, 22p. https://journals.openedition.org/espacepolitique/3543 consulté le 01/06/2018.

RERAT P., SÖDERSTRÖM O., BESSON R., PIGUET É. (2008) « Une gentrification émergente et diversifiée : le cas des villes suisses ", Espaces et sociétés, vol. 1, n 132-133, pp. 39-56. https:// www.cairn.info/revue-espaces-et-societes-2008-1-page-39.htm consulté le 01/06/2018.

RODE S. (2008) «La prévention du risque d'inondation, facteur de recomposition urbaine? L'agglomération de Blois et le déversoir de la Bouillie ». L'Information géographique, vol. 72, $\mathrm{n}^{\circ} 4$, pp. 6-26. https://www.cairn.info/revue-1-information-geographique-2008-4-page-6.htm consulté le $01 / 06 / 2018$.

RODE S., GRALEPOIS M. (2017) « Vers un urbanisme adapté au risque d'inondation? ? in VINET F., (dir.), Floods. Volume 2 risk management, Editions ISTE PRESS \& Elsevier, pp. 365-378.

ROUSSEAU M. (2008) « 'Bringing politics back in' : la gentrification comme politique de développement urbain? Autour des 'villes perdantes' ", Espaces et sociétés, vol. 1-2, n 132, pp. 75-90. https://www.cairn.info/revue-espaces-et-societes-2008-1-page-75.htm consulté le 01/06/2018.

SEMMOUD N. (2005) «Valorisation patrimoniale et changement social : un pléonasme ? in Gravari-Barbas M., Habiter le patrimoine : enjeux, approches, vécu, Rennes, Presse Universitaire de Rennes, pp. 265-280.

SERVICE DE L'AMÉLIORATION DE L'HABITAT DE LA VILLE DU HAVRE (2013), Stratégie d'intervention de la ville du havre en faveur de la requalification de l'habitat privé et Analyse interne de l'évolution des quartiers Sud.

SMITH D.P., BUTLER T. (2007) « Conceptualising the Socio Spatial Diversity of Gentrification: 'To Boldly go' into Contemporary Gentrified Spaces, the 'Final Frontier'? », Environment and Planning A, n 39 (1), pp. 2-9.

SMITH H., GARCIA FERRARI M. S. (2012), Waterfront regeneration: Experiences in city-building, Abingdon, Oxon \& New York: Routledge.

SOCIÉTÉS CONTEMPORAINES (2006) « Gentrification : discours et politiques urbaines (France, Royaume-Uni, Canada) », vol. 3, n 63, pp. 5-13.

STRALE M. (2017) «Quelle place pour les activités portuaires et logistiques à Bruxelles ?», Brussels Studies, n 109, mars 2017, 17p. http://brussels.revues.org/1491 consulté le 01/06/2018.

VAN CRIEKINGEN M. (2008) « Comment la gentrification est devenue, de phénomène marginal, un projet politique global », revue Agone, n 38-39, pp. 71-88.

VELTZ P. (2008), Des lieux et des liens : le territoire français à l'heure de la mondialisation, Paris, Éditions de l'Aube, 176p.

VERDELLI L. (2014) “Port heritage as a material support for port-city's identity in Water and cities, managing a vital relationship", proceedings of the 50th ISOCARP Congress Urban transformations, cities and water, pp. 1424-1434.

VERDELLI L., MORUCCI F. (2014) « Renouveler l'identité de la ville entre culture portuaire et loisirs. Le cas de Livourne », Loisir et Société, vol. 37, n 1, April 2014, pp. 58-78.

VESCHAMBRE V. (2007) «Le processus de patrimonialisation : revalorisation, appropriation et marquage de l'espace », Vox Geographica, 2 novembre 2007, pp. 1-6. http://cafe-geo.net/wpcontent/uploads/processus-patrimonialisation.pdf consulté le 01/06/2018. 
VILLE DU HAVRE (2018), Un territoire en mutation, site officiel de la Ville du Havre.

https://www.lehavre.fr/ma-ville/un-territoire-en-mutation, consulté le 10 avril 2018

\section{NOTES}

1. . La bibliographie en ce qui concerne les waterfronts urbains maritimes et fluviaux est très abondante, elle s'est notamment enrichie au cours des dernières années, en particulier grâce aux travaux de Brown, 2009 ; Desfor, Laidley, et al., 2012 ; Gras, 2010 ; Hein, 2011 ; Prelorenzo, 2010 ; Smith, Garcia Ferrari, 2012.

2. . Michel Lussault dira à propos du Millenium Park à Chicago : « Nous avons là un cas d'action urbanistique majeure, pour laquelle il n'a pas existé de véritable plan initial et qui s'est ajusté en permanence aux contextes : un quasi-modèle de grand projet d'urbanisme flexible, adaptatif et pragmatique » (Lussault, 2013).

3. . Les éléments fondamentaux de la définition contemporaine d'une gentrification pas directement liée à un contexte spatial spécifique, sont, pour Davidson et Lees (2005) : «(1) the reinvestment of capital; (2) the social upgrading of locale by incoming high-income groups; (3) landscape change; and (4) direct or indirect displacement of low-income groups " (Davidson, Lees, 2005)

NDLR: Traduction proposée par les auteurs: «réinvestissement de capital (1), valorisation sociale des habitants locaux par l'arrivée d'habitants à hauts revenus (2), évolution du paysage (3), et déplacement direct ou indirect des populations à bas revenus »

4. . La notion de « risque majeur » se caractérise par la possibilité d'occurrence d'un aléa de faible fréquence mais d'une énorme gravité (nombreuses victimes, dommages importants aux biens et à l'environnement), tels que les risques naturels (avalanche, feux de forêt, inondation...) ou les risques technologiques.

5. . Cet article reprend et complète la communication orale faite lors du colloque « Fabriquer et habiter les villes à l'ère de la mondialisation ", à l'Université Badji Mokhtar d'Annaba en Algérie les 20-21 avril 2015.

6. . Les travaux présentés sont issus du PRojet d'Études sur la Contrainte d'Inondation dans les Espaces Urbains (PRÉCIEU), financé par le programme Risques, Décision, Territoires (RDT 2013) du Ministère de l'Écologie, du Développement Durable et de l'Énergie, novembre 2013-avril 2017 : http://rdt-risques.fr

7. . Orillard Florence, Etude de la contrainte d'inondation dans les espaces urbains. Cas du quartier de l'Eure au Havre, Stage de fin d'études, École Polytechnique Universitaire, Université de Tours, réalisé à l'office Régional des Risques Majeurs de l'Estuaire de la Seine, encadré par Pascal Mallet, juin-septembre 2014. Disponible sur le catalogue de la bibliothèque de l'Université de Tours : http://portail.scd.univ-tours.fr

8. . Iriart Gabriela, Surreaux Hélène, La prise en compte du risque d'inondation par les architectes dans le projet urbain en zone inondable : analyse de la profession d'architecte. Cas du quartier de l'Eure au Havre, Projet de fin d'études, École Polytechnique Universitaire, Université de Tours, 2014. Disponible sur le catalogue de la bibliothèque de l'Université de Tours : http://portail.scd.univtours.fr

9. . Dans le Projet de fin d'études de Gabriela Iriart et Hélène Surreaux, les auteurs ont rencontré six architectes et/ cabinets d'urbanisme qui sont intervenus sur le projet du quartier de l'Eure. Dans le Stage de fin d'études de Florence Orillard, l'auteure a interviewé : la Direction Etudes Urbaines et le Service Urbanisme de la Ville du Havre, la Direction pour l'Information sur les Risques Majeurs et la Direction du Cycle de l'Eau de la Communauté d'Agglomération de la Région Havraise, des chargés d'études de l'Agence d'Urbanisme de la Région Havraise ou encore la Mission ville-port du Grand Port Maritime du Havre. 
10. . En savoir plus sur le site internet de HAROPA (consortium des trois Grands Ports Maritimes du HAvre-ROuen-PAris) : http://www.haropaports.com, notamment sur le tableau statistique de 2017 concernant le Port du Havre : http://www.haropaports.com/sites/haropa/files/ u21/2017_statistiques_definitives_2017.pdf

11. Pour Laurent Davezies, "L'économie résidentielle renvoie à l'offre territoriale non productive, publique ou sociale (revenus des navetteurs, des retraités et des touristes), mesurée en montant monétaires. L'économie présentielle renvoie plutôt à la demande effective sur le territoire, à la consommation (en quelque sorte à la propension locale à consommer) » (Davezies, 2009 : 53).

12. . Pour Le Bart (2011), le marketing territorial est l'ensemble des actions menées par une collectivité afin d'améliorer son image auprès de groupes cibles supposés être en mesure de participer à son développement.

13. Le Grand Port Maritime du Havre est l'établissement public chargé de la gestion du port maritime.

14. . La Directive européenne d'octobre 2007 relative à l'évaluation et la gestion des risques d'inondation a été transposée en droit français dans la loi portant engagement national pour l'environnement de juillet 2010. La directive se décline en stratégies locales comportant notamment de nouveaux scénarii et in fine de nouvelles cartographies. Informations disponibles sur le site de la Préfecture de Seine Maritime :

www.seine-maritime.gouv.fr/Politiques-publiques/Environnement-et-prevention-des-risques/ Risques-technologiques-et-naturels/Territoires-a-Risque-Important-d-Inondation-TRI/TRI-duHavre

15. . Traduction proposée par les auteures «Par son échelle, sa rythmique et ses matériaux, le projet fait partie intégrante d'un paysage portuaire réaménagé, créant la transition entre l'échelle de la maison individuelle et le port dans son ensemble "

16. . Nicolas Cochard, «Les bas-fonds d'une ville portuaire : l'exemple du Havre au prisme de la presse et de la littérature locales ", Médias 19 [En ligne], Enquêtes dans les bas-fonds et le monde criminel, Guillaume Pinson (dir.), Presse, prostitution, bas-fonds (1830-1930), Publications, mis à jour le : 17/05/2013, URL : http://www.medias19.org/index.php?id=13395

17. . Traduction proposée par les auteurs du texte original suivant « to ensure that the conceptual purity of gentrification is not diluted"

18. . Les derniers épisodes de submersion marine des quartiers sud remontent au XIXème siècle.

19. Ces préconisations portent des éléments techniques des installations tels que la hauteur des arrivées d'eau ou la mise en place de valves anti-retour.

20. . Voir le projet du Quai Southampton du Havre par l'architecte urbaniste Michel Desvigne, http://www.lehavre.fr/node/40094

21. . Pour en savoir plus sur le projet Pirmil - Les Isles : http://www.nantes-amenagement.fr/ projet/pirmil-les-isles/

22. . Pour en savoir plus sur le projet Seine-Ardoines : https://www.iau-idf.fr/savoir-faire/nostravaux/edition/amenagement-et-risque-inondation-une-demarche-innovante-en-seineamont.html

23. . Depuis la fermeture des industries dans les années 1970, ces espaces ont été fermés au public. Appartenant à l'État puis vendus depuis 2008 au Grand Port Maritime du Havre, ces espaces ne supportent pas d'usages formels (promenade) ou informels (squat), et les friches portuaires sont inconnues du grand public. 


\section{RÉSUMÉS}

Dans la lignée des réflexions sur les processus de gestion territorialisée des risques naturels, l'article s'attache à montrer comment les contraintes liées à la prévention des inondations sont mobilisées pour poursuivre un objectif de gentrification en rendant nécessaire le renouvellement d'un habitat précaire par du logement de haut standing. Dans le quartier industrialo-portuaire du Havre par exemple, les contraintes liées à la prévention du risque d'inondation viennent offrir une justification incontestable, car formulées en termes de sécurité collective pour in fine construire un quartier délibérément sans mixité sociale. Comment l'expliquer? De plus en plus, les villes sont mises en concurrence, et ceci est d'autant plus vrai pour les villes portuaires telles que Le Havre (France) qui doivent se positionner à une échelle mondiale. Pour se maintenir dans cette compétition, les villes-ports réinterprètent leurs spécificités historiques et territoriales et investissent leur force symbolique dans des projets de quartiers urbains destinés à attirer une population solvable. Le renouvellement urbain du quartier industrialo-portuaire de l'Eure au Havre met en valeur le patrimoine portuaire mais s'accompagne d'une gentrification indirecte de type new build gentrification, sous couvert d'une opération urbaine nécessairement haut de gamme afin de rentabiliser la complexité du projet en zone inondable.

Among the studies on natural risks, spatial transformations and local governance, the paper aims to analyse how flood prevention rules are implemented as political instruments to pursue gentrification, as if flood rules can justify high standing level renewal of old poor areas. Flood prevention rules and measures in some industrial-harbour areas are used to legitimate indirect gentrification. Arguing for collective security and necessity of growth, at the end, the city is strategically developed on flood prone areas. For instance, in Le Havre (France), even if the gentrification is clearly visible, there are no complaints. How can it be explained? Cities are more and more submitted to competitiveness, and even more if they belong to the industrial harbour cities that need to be visible at a worldwide scale. The city of Le Havre is a textbook to illustrate the challenges of declining industrial cities. To stimulate the competitiveness, Le Havre is capitalizing its heritage strengths through new urban projects. These rapidly developing urban areas in industrial-harbour zones bring wealthy new residents and businesses and promote a new face and image of the city, while threatening long-standing poorer residents. These urban projects invoke the heritage of the past maritime tradition, in order to reinvent an attractive landscape. Usually, this indirect gentrification comes together with protest movements, but the integration of risk prevention measures in the resilient urban project seems enough to justify the socio-economic upgrading of the area.

\section{INDEX}

Thèmes : Carnets de recherches

Mots-clés : renouvellement urbain, interface ville-port, risque, inondation, gentrification

Keywords : urban renewal, city-port interface, flood, risk, gentrification 


\section{AUTEURS}

\section{FLORENCE ORILLARD}

Universitaire

Université Toulouse Jean Jaurès

Laboratoire LEREPS EA 4212

florence.orillard@etu.univ-tlse2.fr

\section{MATHILDE GRALEPOIS}

Universitaire,

Département Aménagement et Environnement, École Polytechnique

Université de Tours

Laboratoire CITERES UMR 7324

mathilde.gralepois@univ-tours.fr

\section{LAURA VERDELLI}

Département Aménagement et Environnement, École Polytechnique Universitaire, Université de Tours

Laboratoire CITERES UMR 7324

laura.verdelli@univ-tours.fr 